ESAIM: PROCEEDINGS, April 2012, Vol. 36, p. 73-105

Danièle Fournier-Prunaret, Laura Gardini, \& Ludwig Reich, Editors

\title{
BREAKING THE CONTINUITY OF A PIECEWISE LINEAR MAP
}

\author{
Viktor Avrutin $^{1}$, Michael Schanz ${ }^{1}$ And Björn Schenke ${ }^{1}$
}

\begin{abstract}
Knowledge about the behavior of discontinuous piecewise-linear maps is important for a wide range of applications. An efficient way to investigate the bifurcation structure in 2D parameter spaces of such maps is to detect specific codimension-2 bifurcation points, called organizing centers, and to describe the bifurcation structure in their neighborhood. In this work, we present the organizing centers in the 1D discontinuous piecewise-linear map in the generic form, which can be used as a normal form for these bifurcations in other 1D discontinuous maps with one discontinuity. These organizing centers appear when the continuity of the system function is broken in a fixed point. The type of an organizing center depends on the slopes of the piecewise-linear map. The organizing centers that occur if the slopes have an absolute value smaller than one were already described in previous works, so we concentrate on presenting the organizing centers that occur if one or both slopes have absolute values larger than one. By doing this, we also show that the behavior for each organizing center can be explained using four basic bifurcation scenarios: the period incrementing and the period adding scenarios in the periodic domain, as well as the bandcount incrementing and the bandcount adding scenarios in the chaotic domain.
\end{abstract}

AMS (2000) subject classification. 37E05, 37G10, 37G35.

Keywords. Discontinuous piecewise-linear map, codimension-2 bifurcation, organizing center.

Résumé. Les connaissances sur le comportement d'applications linéaires par morceaux discontinues sont importantes pour de nombreuses applications. Une méthode puissante pour étudier la structure de bifurcation dans les espaces de paramètre $2 \mathrm{D}$ de telles applications est de détecter des points de bifurcation spécifiques de codimension 2, appelés centres organisateurs, et de décrire la structure de bifurcation dans leur voisinage. Dans ce travail, nous présentons les centres organisateurs pour une application linéaire par morceaux discontinue 1D sous forme générique, ce qui peut être utilisé comme une forme normale pour ces bifurcations d'autres applications discontinues 1D avec une discontinuité. Ces centres organisateurs apparaissent lorsque la continuité de la fonction du système est brisée en un point fixe. Le type d'un centre organisateur dépend des pentes de l'application linéaire par morceaux. Les centres organisateurs qui se produisent lorsque les pentes ont une valeur absolue plus petite que un ont déjà été décrits dans des travaux précédents, et nous nous concentrons donc sur la présentation des centres organisateurs qui se produisent si une ou plusieurs pentes ont des valeurs absolues plus grandes que un. Par là même, nous montrons également que le comportement de chaque centre organisateur peut être expliqué en utilisant quatre scénarios élémentaires de bifurcation : les scénarios d'incrément de période et d'addition de période en domaine périodique, et les scénarios d'incrément de bandcount et d'addition de bandcount en domaine chaotique.

Mots clefs. Transformation linéaire par morceaux discontinue, bifurcation de codimension 2 , centre organisateur.

1 IPVS, University of Stuttgart, Universitätstrasse 38, 70569 Stuttgart, Germany; email: Viktor.Avrutin, Michael.Schanz, Bjoern.Schenke@ipvs.uni-stuttgart.de.

(C) EDP Sciences, SMAI 2012 


\section{INTRODUCTION}

Discontinuous piecewise-smooth maps provide an adequate mathematical description of many practical applications ranging from power electronic circuits and A/D converters over micro mechanical systems, impacting and friction dominated systems with stick-slip behavior up to control and communication systems (see [1-3] for references). Moreover, it is in the meanwhile well-known that Poincaré return maps of smooth flows showing lowdimensional chaos (including the most popular example in this area, namely the Lorenz flow) are non-smooth and discontinuous [4-6].

To understand possible behaviors of these systems and to achieve a desired behavior via a suitable system design, it is necessary to understand the bifurcation structures occurring in multi-dimensional parameter spaces. The most efficient way to investigate these structures is to detect their organizing centers, which are given by bifurcations of higher codimension $[7,8]$. Typically, at such a point in the parameter space an infinite number of existence regions of periodic orbits emerges. In the case that these orbits are stable, their stability regions may cover an extended part of the parameter space and hence explain several 1D bifurcation scenarios observed in parts of the parameter space located far away from the organizing center [9]. Organizing centers can also lead to the appearance of unstable periodic orbits. These can turn stable when parameters are varied and they can induce a bifurcation structure consisting of crisis bifurcations within the robust chaotic domain (for the definition of robustness for chaotic attractors we refer to [10]). So, regardless of the stability of appearing orbits, it is worthwhile to investigate each type of organizing center thoroughly.

Our work is motivated by the following observation. Typically, organizing centers occur at points in parameter space where the map (or some composite map consisting of parts of several iterated functions) becomes continuous. To understand the properties of the organizing centers that occur when the map becomes continuous, we have to study the regions in parameter space around these points, which means breaking the continuity.

In this work we consider the most easy case of discontinuous systems, namely the 1D piecewise linear map with one discontinuity given in the general form by

$$
x_{n+1}=f\left(x_{n}\right)=\left\{\begin{array}{lll}
f_{\ell}\left(x_{n}\right)=a_{\ell} x_{n}+\mu_{\ell} & \text { if } & x_{n}<0 \\
f_{r}\left(x_{n}\right)=a_{r} x_{n}+\mu_{r} & \text { if } & x_{n}>0
\end{array}\right.
$$

Note that the map is intentionally not defined at $x=0$, as the value $f(0)$ is not important ${ }^{1}$, except for the behavior at the bifurcation points (depending on a particular application, the definition at the boundary may be either $f(0)=f_{\ell}(0)$ or $f(0)=f_{r}(0)$ ). As one can immediately see, in general (for $\mu_{\ell} \neq \mu_{r}$ ) the map is discontinuous. However, at the point $\mu_{\ell}=\mu_{r}=0$ the map is continuous and has a fixed point at the boundary $x=0$. Therefore, it is natural to ask the following question: what happens when the continuity of the function at this fixed point is broken, that means the offsets $\mu_{\ell}$ and $\mu_{r}$ are varied from $\mu_{\ell}=\mu_{r}=0$ to a value for which the map is discontinuous. As we shell see below, the results of this continuity breaking are essentially different depending on the slopes $a_{\ell}$ and $a_{r}$, for which we have to distinguish between the following three generic cases. The map can be increasing on both sides $\left(a_{\ell}, a_{r}>0\right)$ of the point $x=0$, it can be decreasing on both sides $\left(a_{\ell}, a_{r}<0\right)$ and it can be decreasing on one side, and increasing on the other $\left(a_{\ell}>0, a_{r}<0\right.$ or $\left.a_{\ell}<0, a_{r}>0\right)$. Note that, in the third case, it does not really matter on which side the map is chosen to be increasing and on which to be decreasing, since both variants are conjugate to each other.

Until now, the breaking of the continuity at a fixed point was investigated for a few particular cases. In [11] it is proved that if a piecewise smooth map is increasing and contractive on both sides of the boundary, then the point at which the function is continuous represents an organizing center of the period adding ${ }^{2}$ type. For the

\footnotetext{
${ }^{1}$ By contrast, the limit values $f_{\ell}(0)=\mu_{\ell}$ and $f_{r}(0)=\mu_{r}$ are crucial for the dynamics.

${ }^{2}$ It is worth to emphasize that the notation "period adding" is used in the literature not uniquely but for many different bifurcation scenarios. In our work, we distinguish between the period adding and period incrementing scenarios. The term period adding refers to the self-similar bifurcation scenario in which the periodicity regions (sometimes referred to as mode-locking tongues or Arnold's tongues) are located in the parameter space according to the Farey addition rule for the rotation numbers of the corresponding periodic orbits. More precisely, between two periodicity region associated with the rotation numbers $\frac{u_{1}}{v_{1}}$ and $\frac{u_{2}}{v_{2}}$
} 
increasing/decreasing case in was proved in [12] that if the map is contractive on both sides of the boundary, then the point at which the function is continuous represents an organizing center of the period incrementing type, formed by the in general pairwise overlapping existence regions of the basic orbits $O_{\mathcal{R} \mathcal{L}^{n}}$. This result was extended in [13], where a different proof is given, showing that if the map is in the increasing/decreasing configuration, but not necessarily contractive, then the existence regions of the same basic orbits are originating from the point at which the function is continuous. However, the orbits are not necessarily stable in this case, and the bifurcation structure involving the unstable orbits is not investigated in the cited work.

Note that the organizing centers of the period adding and period incrementing type are so-called big bang bifurcations. These are bifurcation points in parameter space with a codimension larger than one, which have the property that an infinite number of bifurcation curves are issuing from that point. Most of the organizing centers presented in this work are big bang bifurcations.

We are asking now the question which organizing centers appear if we do not restrict ourselves to the contracting case. Preliminary investigations of this have shown what appears to be a great variety of different bifurcation scenarios. The intention of this work is, besides showing the different possible scenarios, to demonstrate that this variety of scenarios is not as complicated as it seems at first glance. Instead, there are a few basic scenarios, which appear several times in different combinations and configurations. In the present work, we restrict ourselves to showing the cases which are possible in piecewise linear maps, keeping however in mind that piecewise-linear maps can be used as an approximation for other piecewise-smooth systems. Therefore we are confident that the organizing centers discussed below appear in other piecewise-smooth systems as well. It is worth to emphasize that in the present work we do not intend to determine the conditions leading to appearance of these organizing centers. In fact, to find a rigorous proof for each of them represent a challenging task which we leave for future research. For some cases these proofs are already established (in these cases the corresponding references are given below), while for other cases the conditions are still unknown. The aim of our work is much less ambitious: we are only reporting different organizing centers resulting from the breaking of the continuity of the function at its fixed point. In this sense our work represents a preliminary step required for a more deep investigation of the organizing centers. In order to determine the conditions leading to the appearance of each specific type of organizing centers, we need first to identify these types. Moreover, our work is aimed to contribute to the solution of the following problem. It is already known (see for example $[14,15]$ ) that in discontinuous maps border collision bifurcation curves of two periodic orbits may intersect and that the intersection point (a codimension-2) bifurcation may represent a kind of organizing center. It can be shown that the problem in this case can be reduced to the problem of the continuity breaking at a fixed point. Hence, our work will not present a solution to the question what happens at such an intersection, but it represents a preliminary step towards this solution.

This work is structured as follows. In $\S 2$, after the introduction, the method of investigating the organizing centers for varying slopes is explained. The remaining sections follow the three major variations of the slopes. First, $\S 3$ shows several organization centers for the increasing/increasing case. Then, $\S 4$ shows the increasing/decreasing case. Finally, $\S 5$ shows the decreasing/decreasing case.

\section{MODE OF OPERATION}

As system (1) has four parameters, there are six different possibilities to choose a parameter plane to investigate. From these options, we choose the $\left(\mu_{r}, \mu_{\ell}\right)$-plane. This plane has the advantage, that the organizing center, which is investigated in this work, is always located at $(0,0)$. At this point, both fixed points of the system given below by Eq. 3 collide with the border $x=0$, and the map is continuous. Additionally, the $\left(\mu_{r}, \mu_{\ell}\right)$-plane has the advantage of being very easy to describe since all bifurcation curves in the $\left(\mu_{r}, \mu_{\ell}\right)$-plane

there exists a periodicity region with the rotation numbers $\frac{u_{3}}{v_{3}}=\frac{u_{1}}{v_{1}} \oplus \frac{u_{2}}{v_{2}}=\frac{u_{1}+u_{2}}{v_{1}+v_{2}}$. By contrast, the period incrementing scenario is used for a cascade (in the non-degenerate case pairwise overlapping) periodicity regions, whereby the period in each next region is equal to the period in the previous one increased by some constant value: $p_{n+1}=p_{n}+\Delta p=p_{0}+n \Delta p$. 
are straight lines intersecting at the origin. The explanation for that is straight-forward. Every border collision bifurcation and every homoclinic bifurcation curve in system (1) can be described by a term of the form $\left\{\left(a_{\ell}, a_{r}, \mu_{\ell}, \mu_{r}\right) \mid \frac{\mu_{\ell}}{\mu_{r}}=\frac{p_{1}\left(a_{\ell}, a_{r}\right)}{p_{2}\left(a_{\ell}, a_{r}\right)}\right\}$ with $p_{1}$ and $p_{2}$ being polynomials depending on the slopes ${ }^{3}$. If $a_{\ell}$ and $a_{r}$ are fixed, the resulting equation $\frac{\mu_{\ell}}{\mu_{r}}=$ const describes a straight line in the $\left(\mu_{r}, \mu_{\ell}\right)$-plane issuing from the origin $\mu_{\ell}=\mu_{r}=0$. If there is more than one bifurcation curve issuing from the origin (and this is always the case, due to the border collision bifurcations of the fixed point explained below), it is called an organizing center. When moreover there are infinitely many lines issuing from the origin, it is called a big bang bifurcation. In summary, this means that to completely describe the bifurcation scenario of the $\left(\mu_{r}, \mu_{\ell}\right)$-plane, it is sufficient to describe some neighborhood of the origin.

In this work, this is done by varying $\mu_{\ell}$ and $\mu_{r}$ along a circle of radius 1 around the origin. This means that all bifurcations in a $\left(\mu_{r}, \mu_{\ell}\right)$-plane can be described by a variation of the angle $\phi_{\mu}$ between 0 and $2 \pi$ :

$$
\mu_{r}=\cos \left(\phi_{\mu}\right), \quad \mu_{\ell}=\sin \left(\phi_{\mu}\right)
$$

Fig. 1a demonstrates this by showing an example for a 2D bifurcation diagram. The straight lines represent bifurcation curves, the black circle represents the values of $\mu_{\ell}$ and $\mu_{r}$ that are shown in the 1D-bifurcation diagram in Fig. 1b. In this bifurcation diagram one can easily see the existence regions of the fixed points of system (1). These fixed points are given by

$$
x^{\mathcal{L}}=-\frac{\mu_{\ell}}{a_{\ell}-1}, \quad x^{\mathcal{R}}=-\frac{\mu_{r}}{a_{r}-1}
$$

with $x^{\mathcal{L}}$ being the fixed point corresponding to $f_{\ell}$ and $x^{\mathcal{R}}$ being the fixed point corresponding to $f_{r}$. If the offsets are varied along a circle as shown in Eq. (2), each fixed point exists in an interval of length $\pi$. The location of each interval depends on the values of the slopes. Therefore, the fixed point $x^{\mathcal{L}}$ exists if $a_{\ell}>1$ and $\mu_{\ell}>0$ or $a_{\ell}<1$ and $\mu_{\ell}<0$. The fixed point $x^{\mathcal{R}}$ exists if $a_{r}>1$ and $\mu_{r}<0$ or $a_{r}<1$ and $\mu_{r}>0$. The fixed point $x^{\mathcal{L}}$ is stable if $\left|a_{\ell}\right|<1$ and $x^{\mathcal{R}}$ is stable if $\left|a_{r}\right|<1$. Note that to demonstrate the whole picture of the system's behavior, the range of the state variable $x$ in the bifurcation diagrams has been scaled with the topology-preserving mapping:

$$
\mathcal{S}(x):(-\infty, \infty) \mapsto\left(-\frac{\pi}{2}, \frac{\pi}{2}\right), \quad \text { with } \quad \mathcal{S}(x)=\arctan (x) .
$$

which allows to show graphically the values from $-\infty$ to $\infty$. Therefore, throughout this paper the meaning of the labels on the horizontal and vertical axes in the bifurcation diagrams is different. On the horizontal axis the labels refer to angles, as shown in Fig. $1 a$, while on the vertical axis they refer to the values of the state variable scaled by (4) (see Fig. 1b). Not shown in Fig. 1 is the period diagram corresponding to the bifurcation diagram. The figures shown later in this work all include such a period diagram, in which the periods $p$ of the stable periodic orbits are shown. Additionally, for multi-band chaotic attractors the number $\mathcal{K}$ of their connected components (bands) is shown in the same diagram. Note also that the parts of the bifurcation and period diagrams corresponding to divergent behavior for all typical initial conditions are shown gray.

In the following sections Figs. 3 - 16 are calculated similar to Fig. 1 and show the the bifurcation structure in the $\left(\mu_{r}, \mu_{\ell}\right)$ parameter plane for a given combination of the slopes $a_{\mathcal{L}}, a_{\mathcal{R}}$, the corresponding shape of the system function, as well as the bifurcation and the period diagrams along the circle around the origin of the $\left(\mu_{r}, \mu_{\ell}\right)$ parameter plane, as given by Eq. (2).

\footnotetext{
${ }^{3}$ To explain this, recall that to calculate the curve of a border collision bifurcation one has to solve the equation $f^{n}(0)=0$ for a suitable branch of the $n$th iterate of $f$. Due to the fact that the functions $f_{\ell}$ and $f_{r}$ are linear (or, more precisely, affine), it can be shown that each branch of $f^{n}$ represents a polynomial with respect to $a_{\ell}, a_{r}$ and is linear with respect to $\mu_{\ell}, \mu_{r}$ and $x$. Therefore, the condition $f^{n}(0)=0$ leads to the equation $p_{1}\left(a_{\ell}, a_{r}\right) \mu_{r}-p_{2}\left(a_{\ell}, a_{r}\right) \mu_{\ell}=0$. The calculation of homoclinic bifurcation is more complicated leads to similar results as well.
} 

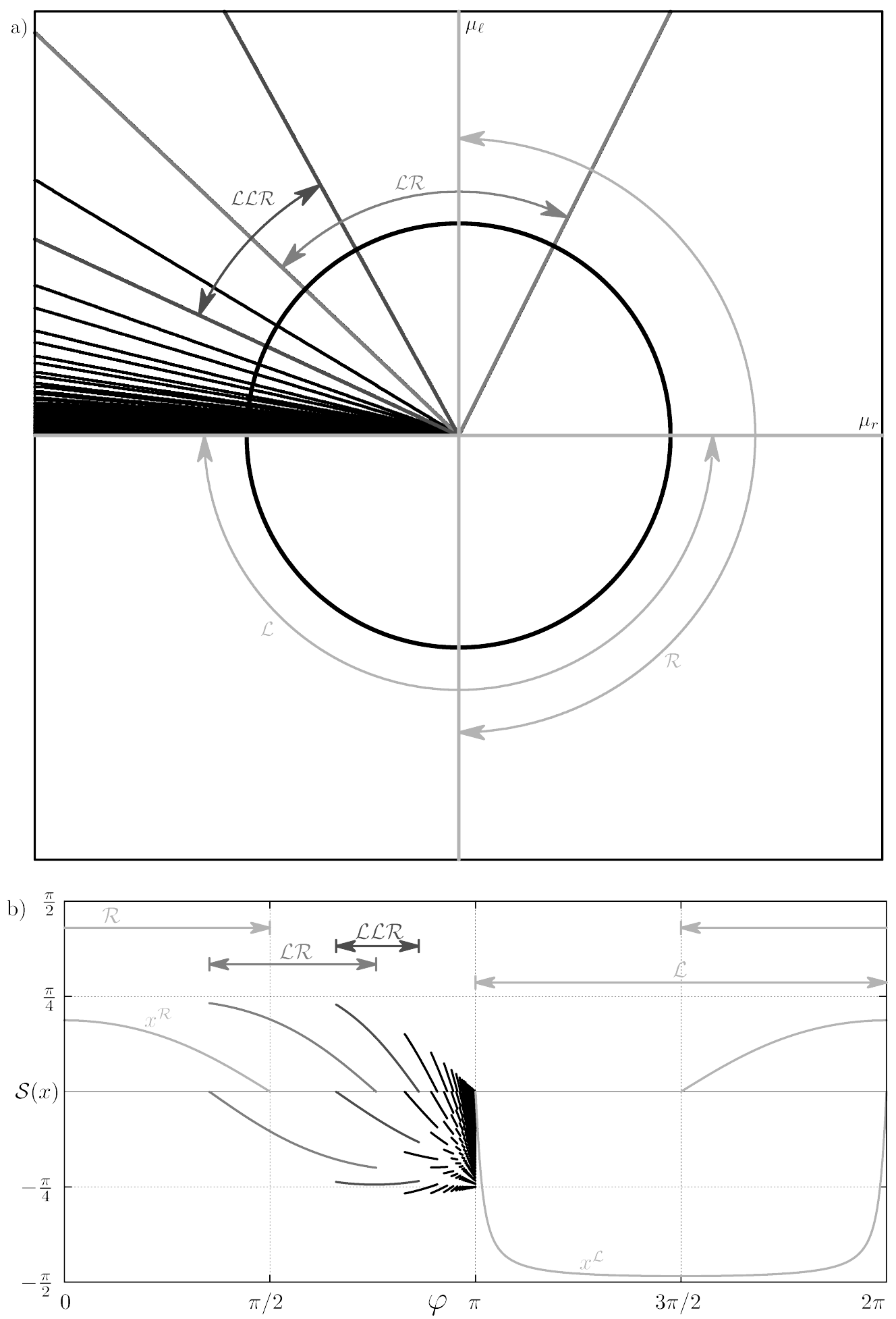

FiguRE 1. a) $\left(\mu_{r}, \mu_{\ell}\right)$-plane for $a_{\ell}=0.95, a_{r}=-0.5$. b) Bifurcation diagram calculated along the black circle in a) 


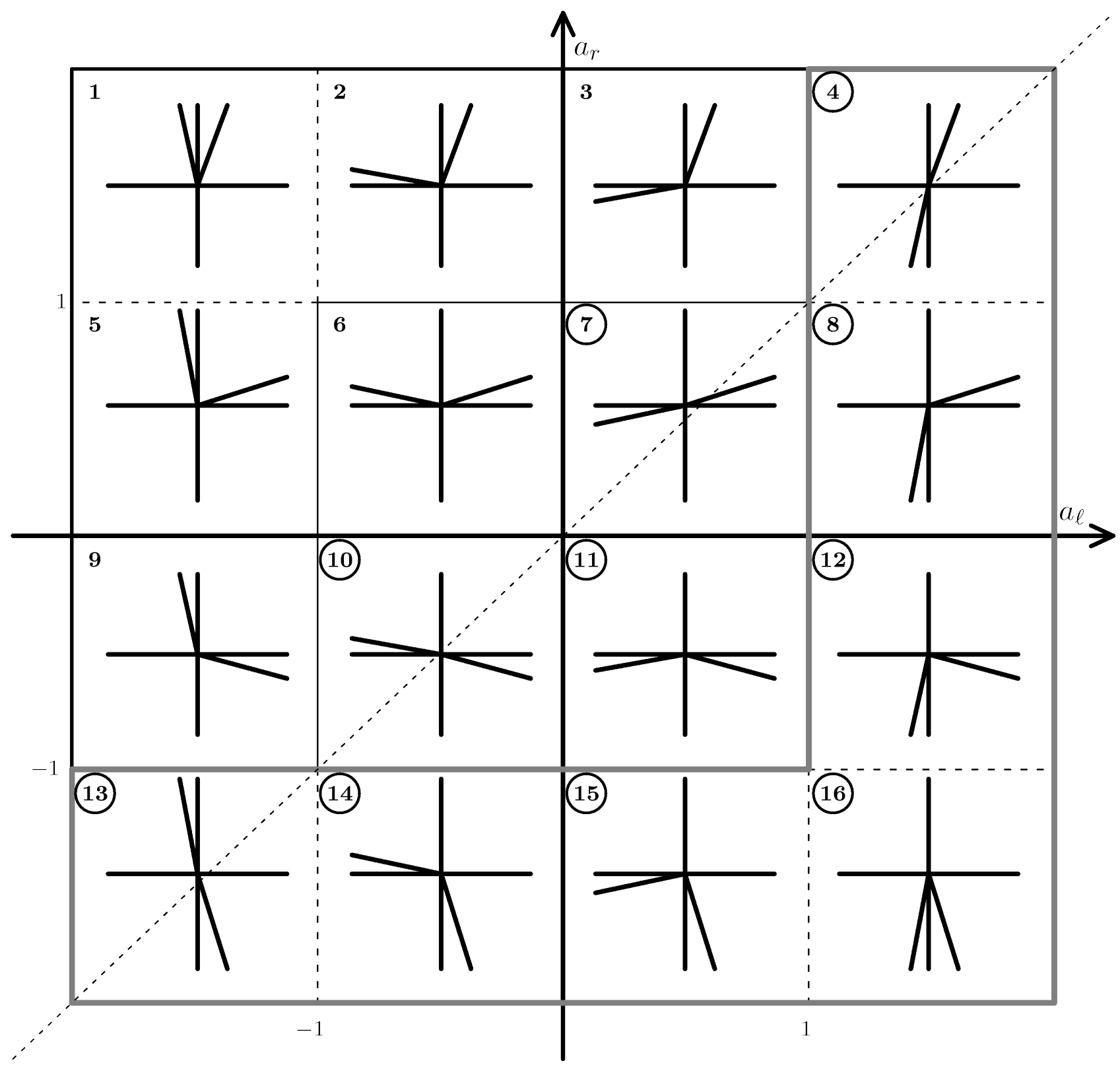

FIGURE 2. Varying the values of the slopes leads to 16 different configurations of the map. The cases which are presented in this work are marked with circles around the numbers. Additionally, the cases with at least one slope above one and which are presented in this work are marked with a gray border.

As was already mentioned in the introduction, our description of the point $\left(\mu_{r}, \mu_{\ell}\right)=(0,0)$ of system (1) follows the 16 different configurations of the slopes (see Fig. 2). Note that a similar approach was already used in [16] and many of the cases reported in the present work were already partially described in that publication. However, as our work is focused on the investigation of $\left(\mu_{r}, \mu_{\ell}\right)=(0,0)$, we prefer to consider the bifurcation scenarios along the circles around the origin in the $\left(\mu_{r}, \mu_{\ell}\right)$ plane, instead of lines in a parameter plane defined by 
an offset and the width of the discontinuity in the system function. This allows us to determine all numerically detectable dynamics possible for each particular choice of the slopes in a much more easy way than it can be done by the approach used in the cited work. In fact, below we report several bifurcation structures not mentioned there.

Since system (1) was already thoroughly investigated for the case that the absolute values of both slopes are smaller than one, we concentrate on cases where at least one slope has an absolute value larger than one. Since slopes can be positive and negative, this means that there are, in principle, $16-4$ cases to consider. However, not all of these cases need to be investigated, due to symmetries inherent in system (1). Two systems which correspond to points in the $\left(a_{\ell}, a_{r}\right)$-plane, which are symmetrical with respect to the line $a_{\ell}=a_{r}$ show the same bifurcation scenarios. The most important effect of this was already pointed out above, since it means that the increasing/decreasing case and the decreasing/increasing case are conjugate to each other. For the increasing/increasing and decreasing/decreasing cases, it means that if only one slope has an absolute value larger than one, only one of the two alternatives needs to be considered. This leaves seven cases to investigate, which are highlighted in Fig. 2 (cases 4, 8, 12,13,14,15 and 16). All main variations of the configuration of the slopes are shown in the figure, with the ones which will be investigated marked with a gray border. Note that also three of the four already investigated cases with both slopes smaller than one will be presented, for sake of completeness (cases 7,10 and 11). The following sections describe the different organizing centers for different combinations of the slopes.

\section{INCREASING/INCREASING CASE}

\subsection{No slope larger than 1}

If both slopes are positive and smaller than one (case 7 in Fig. 2), there will be stable periodic orbits or fixed points for all offsets. If both fixed points exist (in the fourth quadrant of the $\left(\mu_{r}, \mu_{\ell}\right)$-plane), every initial condition within their respective partition will converge to them. If only one of them exists (in the first and the third quadrant), it is globally attractive.

If the fixed points do not exist, there exists a globally attractive absorbing interval $\left[f_{r}(0), f_{\ell}(0)\right]$ (an interval to which every initial condition will be mapped in finite time) for the offsets between the existence regions of the fixed points, on which the system function is always invertible. It was proved in [17] that in this case no unstable orbits and hence also no chaotic behavior is possible. The stable orbits are organized by the period adding scenario (for the proof we refer to $[11,18]$ ). This means that the complete parameter space is structured according to the Farey-tree like period adding scheme: Between the existence regions of an orbit with period $p_{n}$ and an orbit with the period $p_{n+1}$ lies the existence region of an orbit with the period $p_{n}+p_{n+1}$. The symbolic sequences corresponding to the orbits with periods $p_{n}$ and $p_{n+1}$ are concatenated to form the sequence corresponding to the third orbit with the period $p_{n}+p_{n+1}$. This repeats ad infinitum, so that between the orbits with period $p$ and $p_{n}+p_{n+1}$ an orbit with period $2 p_{n}+p_{n+1}$ can be found, as well as orbits with periods $3 p_{n}+p_{n+1}, 4 p_{n}+p_{n+1}$, and so on. This way a description of the self-similar structure of period adding is generated.

Fig. 3 shows the scenario with both slopes being equal. Choosing slopes which are not equal leads to the same scenario consisting of the same periodic orbits. The only difference is that the existence regions of the particular orbits change in size with some getting smaller and some getting larger.

\subsection{One slope larger than 1}

If one slope is set to a value larger than one (case 8 in Fig. 2) there may exist a stable (but not globally attracting) fixed point, stable periodic orbits, chaos and divergence, depending on the offsets. Note that, when referring to divergence in this work, we mean that all typical initial conditions diverge. We do not say anything about the behavior of the system for atypical initial conditions. This case is depicted in Fig. 4.

For every set of positive slopes with one of them larger than one, there exists a combination of offsets for which the system function is not invertible on the absorbing interval $\left[f_{r}(0), f_{\ell}(0)\right]$. This means that not only 
stable periodic dynamics are possible, but also chaos (however, not the coexistence of both. For a proof of that, see [17]). The condition for the system function to be invertible on the absorbing interval is $f_{\ell}\left(f_{r}(0)\right)>f_{r}\left(f_{\ell}(0)\right)$, which can be used to calculate the boundary separating the periodic and chaotic domains, $\tau$ :

$$
f_{\ell}\left(f_{r}(0)\right)=f_{r}\left(f_{\ell}(0)\right) \Rightarrow \tau:=\left\{\left(a_{\ell}, a_{r}, \mu_{\ell}, \mu_{r}\right) \mid \frac{\mu_{\ell}}{\mu_{r}}=\frac{a_{\ell}-1}{a_{r}-1}\right\}
$$

As long as the system function is invertible on the absorbing interval, there will be stable orbits organized by period adding. The interval in which this is the case is bordered on one side by the stable fixed point and by $\tau$ on the other. On the other side of $\tau$ the system function is not invertible on the absorbing interval. The periodic orbits there are not stable. These unstable orbits are still organized by the adding scheme between an unstable orbit with period $p_{n}$ and an unstable orbit with period $p_{k}$ is an unstable orbit with period $p_{n+k}$. Instead of period adding, the interval is organized by the so-called bandcount adding scenario introduced in [19], see also [20]. In short, the bandcount adding scenario can be summarized as follows (for a more detailed description, we refer to [19]). For most parameter values the unstable periodic orbits will be contained in the chaotic attractor. However, at certain parameter values, an unstable periodic orbit exists outside the chaotic attractor and the attractor is split into several intervals (also called connected components or bands). The number of these intervals is the so-called bandcount of the attractor. Orbits which exist outside the attractor at the boundary of its immediate basin can collide with the attractor at a crisis bifurcation, which causes typically a change in the bandcount. A periodic orbit with period $p$ located at the boundary of the immediate basin of the attractor causes a bandcount of at least $\mathcal{K}=p+1$. When chaotic attractors are organized by bandcount adding, this means that between a bandcount of $p_{n}+1$ and a bandcount of $p_{n+1}+1$, a bandcount of $p_{n}+p_{n+1}+1$ will be located.

Each periodic orbit is either involved in the period adding (if it is stable) or in the bandcount adding scenario (if it is unstable. Hereby we mean both situations, namely that an orbit is located inside the chaotic attractor, or outside). For a specific orbit, this can be determined by counting the symbols $\mathcal{L}$ and $\mathcal{R}$ in its sequence. An orbit with $m$-times the symbol $\mathcal{L}$ and $k$-times the symbol $\mathcal{R}$ is stable and belongs to period adding if $\left|a_{\ell}^{m} a_{r}^{k}\right|<1$. To give an example, for the values $a_{\ell}, a_{r}$ for which the scenario is shown in Fig. 4 one can see immediately that the basic orbits of the form $O_{\mathcal{R} \mathcal{L}^{n}}$ are necessarily unstable. For the basic orbits of the form $O_{\mathcal{L} \mathcal{R}^{n}}$ one can calculate that they are stable for all $n \geq 2$.

Apart from the stable and chaotic intervals, there will also be an interval of divergence. Depending on which side the unstable fixed point is, an offset can always be found so that $x=0$ is mapped to the other side of the unstable fixed point (meaning that $f_{r}(0)<x^{\mathcal{L}}$ or $f_{\ell}(0)>x^{\mathcal{R}}$, respectively). In this case, there will be no absorbing interval and almost all initial conditions will diverge. The border between bandcount adding and divergence is given by the collision of the unstable fixed points with the border of the attractor in a boundary crisis (also called final bifurcation). Depending on the colliding fixed point this means that:

$$
\begin{aligned}
& f_{\ell}(0)=x^{\mathcal{R}} \quad \Rightarrow \quad \eta_{\ell}:=\left\{\left(a_{\ell}, a_{r}, \mu_{\ell}, \mu_{r}\right) \mid \frac{\mu_{\ell}}{\mu_{r}}=-\frac{1}{a_{r}-1}\right\} \\
& f_{r}(0)=x^{\mathcal{L}} \quad \Rightarrow \quad \eta_{r}:=\left\{\left(a_{\ell}, a_{r}, \mu_{\ell}, \mu_{r}\right) \mid \frac{\mu_{\ell}}{\mu_{r}}=1-a_{\ell}\right\}
\end{aligned}
$$

Note that the unstable periodic orbits of the two adding scenarios still exist in the interval of divergence, but they have stopped influencing the dynamic behavior apart from atypical initial conditions.

\subsection{Both slopes larger than 1}

If both slopes are set to values larger than one (case 4 in Fig. 2), the system function will always be not invertible on the absorbing interval. All periodic orbits are unstable and there are chaotic attractors which are structured by the bandcount adding scenario. Similar to the case of one slope smaller than one, the bifurcation between the region with chaotic intervals and divergence can be determined by the collision of the fixed points 
with the boundary of the chaotic attractor. One border is given by $\eta_{\ell}$ (see Eq. (6)) and the other border is given by $\eta_{r}$ (see Eq. (7)). This means that those regions of parameter space which satisfy the conditions $f_{\ell}(0)<x^{\mathcal{R}}$ and $f_{r}(0)>x^{\mathcal{L}}$, will contain chaotic attractors. This case is depicted in Fig. 5.

Note that the slopes can be chosen so that there will be no chaotic intervals and only divergence. The reason for this is easily explained. The condition $f_{\ell}(0)<x^{\mathcal{R}}$ can be written as $\frac{\mu_{\ell}}{\mu_{r}}<-\frac{1}{a_{r}-1}$ and the condition $f_{r}(0)>x^{\mathcal{L}}$ can be written as $\frac{\mu_{\ell}}{\mu_{r}}>1-a_{\ell}$. If the slopes are chosen so that $1-a_{\ell}>-\frac{1}{a_{r}-1}$, one of the conditions will always be false. Consequently, the border between the case for which chaos is possible and the case that there is only divergence can be determined as

$$
1-a_{\ell}=-\frac{1}{a_{r}-1} \Rightarrow\left\{\left(a_{\ell}, a_{r}\right) \mid a_{\ell}=\frac{a_{r}}{a_{r}-1}\right\}
$$



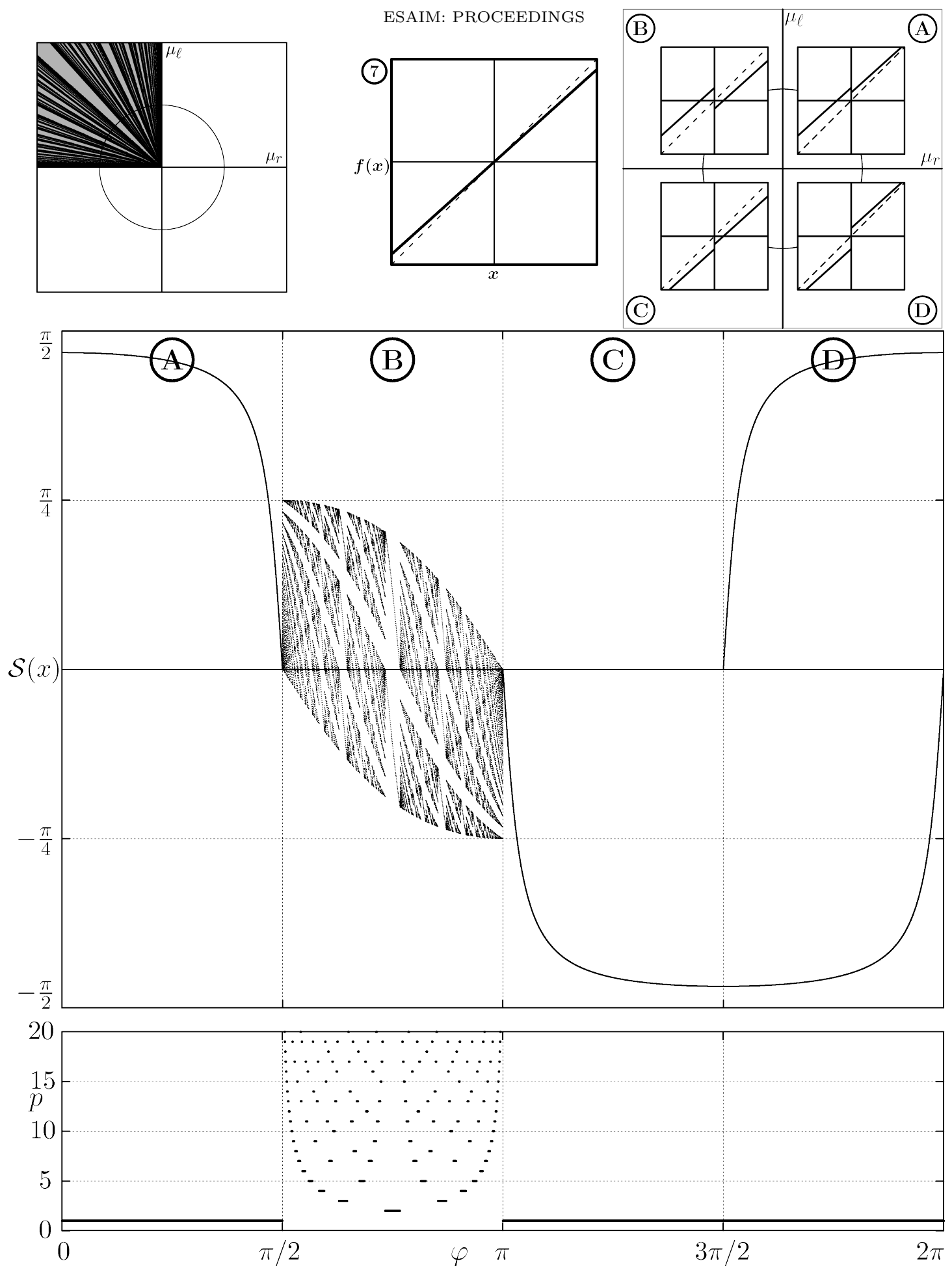

FiguRE 3. Increasing / increasing case with $0<a_{\mathcal{L}}<1,0<a_{\mathcal{R}}<1$.

The second quadrant of the $\left(\mu_{r}, \mu_{\ell}\right)$ plane is filled with the period adding structure, in the remaining three quadrants the fixed points are stable (in the fourth quadrant they coexist $).\left(a_{\mathcal{L}}=0.9, a_{\mathcal{R}}=0.9\right)$ 


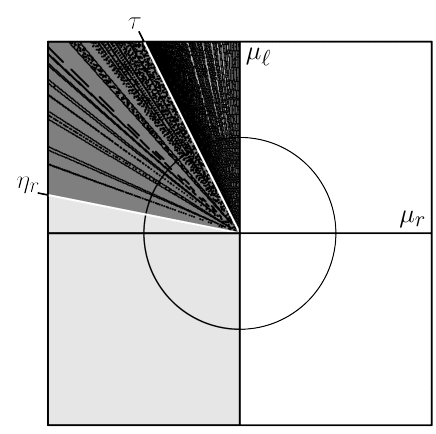

(B)
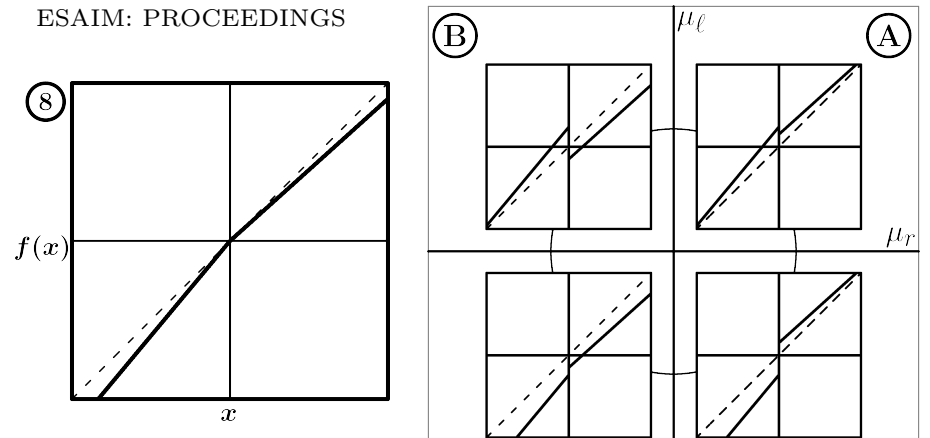

(A)

(C)

(D)
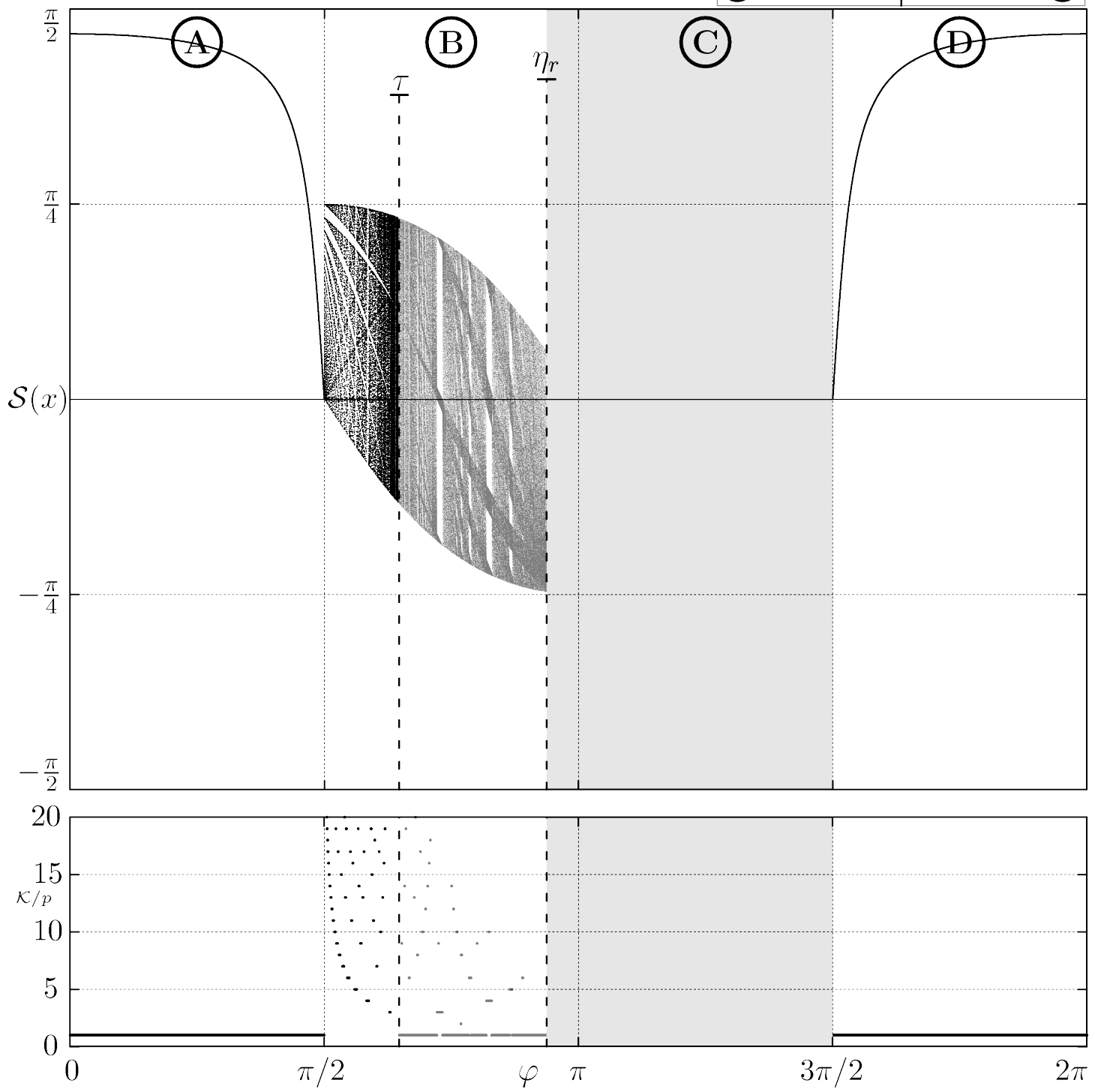

FiguRE 4. Increasing / increasing case with $a_{\mathcal{L}}>1,0<a_{\mathcal{R}}<1$.

In the second quadrant of the $\left(\mu_{r}, \mu_{\ell}\right)$ plane not only the period adding structure but also chaotic attractors organized by the bandcount adding structure exist. In the remaining three quadrants there is a stable fixed point and divergence. $\left(a_{\mathcal{L}}=1.2, a_{\mathcal{R}}=0.9\right)$ 


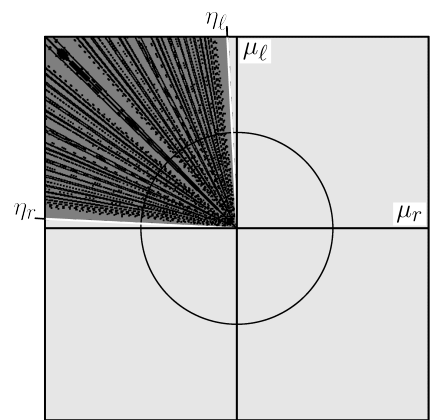

(B)
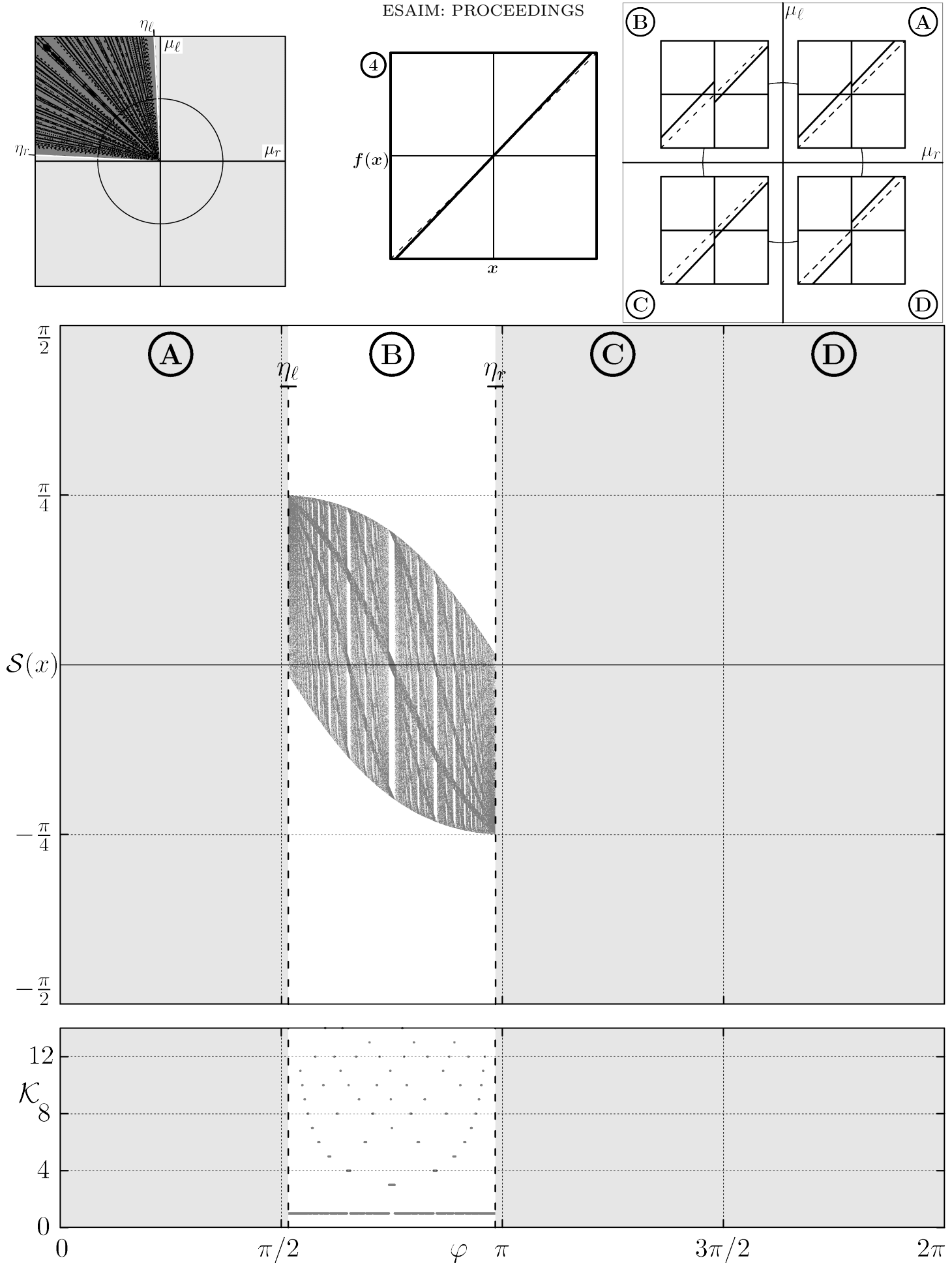

FIGURE 5. Increasing / increasing case with $a_{\mathcal{L}}>1, a_{\mathcal{R}}>1$.

The dynamics outside the second quadrant is divergent, in the second quadrant there is a chaotic domain organized by the bandcount adding structure. $\left(a_{\mathcal{L}}=\right.$ $\left.1.05, a_{\mathcal{R}}=1.05\right)$ 


\section{InCREASING/DECREASING CASE}

\subsection{No slope larger than 1}

If one slope is increasing, the other one decreasing and both have an absolute value less than one (case 11 in Fig. 2), there will be stable periodic orbits or fixed points for all offsets. If both of the fixed points exist, every initial condition within their respective partition will converge to them. If only one of them exists, it is globally attractive.

If the fixed points do not exist, there exists a globally attractive absorbing interval $\left[f_{r}\left(f_{\ell}(0)\right), f_{\ell}(0)\right]$ between the existence regions of the fixed points. The stable orbits existing in the absorbing interval are proved to be [12] organized by the period incrementing scenario. This means that after the existence region of a periodic orbit with the period $p_{n}$ follows the existence region of a periodic orbit with the period $p_{n+1}=p_{n}+1$. These two regions overlap, so that the orbits with period $p_{n}$ and $p_{n+1}$ pairwise coexist for certain parameter values. This is always an infinite sequence which leads to arbitrary high periods in the neighborhood of an accumulation point, which is given by the border collision of the fixed point $x^{\mathcal{L}}$ (see Fig. 6). The orbits forming this bifurcation scenario correspond to the symbolic sequences $O_{\mathcal{R} \mathcal{L}^{n}}$.

\subsection{Increasing slope larger than 1}

If the increasing slope is set to a value larger than one (case 12 in Fig. 2) there may exist the stable fixed point $x^{\mathcal{R}}$, stable periodic orbits, chaos and divergence, depending on the offsets.

If the fixed point $x^{\mathcal{R}}$ does not exist, and there is no divergence there is a family of stable periodic orbits $O_{\mathcal{R} \mathcal{L}^{n}}$ organized by the period incrementing scenario, as proved by Theorem 1 in [13]. These orbits are stable up to a specific value of $n$ and all orbits beyond that are unstable (for example, in Fig. 7, $n=3$ ). This specific value of $n$ is the largest value which satisfies the stability condition $\left|a_{\ell}^{n} a_{r}\right|<1$. The condition can easily be derived using the corresponding iterate $f_{\ell}^{n} \circ f_{r}$. The case in which there exists an $n$ so that $\left|a_{\ell}^{n} a_{r}\right|=1$ represents a particular case. Since we know that $a_{\ell}>1$, the value $\left|a_{\ell}^{n} a_{r}\right|$ increases monotonically with $n$. This means that the periodic orbit $O_{\mathcal{R} \mathcal{L}^{n+1}}$, which follows the periodic orbit $O_{\mathcal{R} \mathcal{L}^{n}}$ with the largest $n$ that still satisfies $\left|a_{\ell}^{n} a_{r}\right|<1$ must be unstable. The orbit $O_{\mathcal{R} \mathcal{L}^{n+1}}$ is followed by the rest of the orbits of the family $O_{\mathcal{R} \mathcal{L}^{m}}$ (with $m>n+1$ ), which must be all unstable as well. This means that the border collision bifurcation at which the orbit corresponding to the highest value of $n$ stops to exist corresponds to a stability border. This border has the property that on one side of it, all periodic orbits are unstable, while on the other side stable periodic orbits exist (in Fig. 7 the stability border is marked with $\left.\xi_{\mathcal{R} \mathcal{L}^{3}}\right)$.

On the other side of the stability border chaos exists, which is organized by the so-called bandcount increment scenario. In a way, the basic structure of bandcount incrementing is similar to period incrementing. If there is a region in parameter space with bandcount $p$, there will be a region near to it, with bandcount $p+1$. However, depending on parameter values, it is also possible that these regions overlap, which increases the bandcount to $2 p$. Besides this basic structure bandcount incrementing often includes complicated substructures, containing regions with different bandcounts. For a detailed description of bandcount incrementing see [21].

The border between chaos and divergence is given by the collision of the unstable fixed point $x^{\mathcal{L}}$ with the border of the attractor in a boundary crisis, which is determined by

$$
f_{r}\left(f_{\ell}(0)\right)=x^{\mathcal{L}} \Rightarrow \eta_{\ell r}:=\left\{\left(a_{\ell}, a_{r}, \mu_{\ell}, \mu_{r}\right) \mid \frac{\mu_{\ell}}{\mu_{r}}=-\frac{a_{\ell}-1}{a_{\ell} a_{r}-a_{r}+1}\right\}
$$

After $\eta_{\ell r}$ there is no absorbing interval, and all typical initial conditions diverge.

Next to the border between chaos and divergence $\eta_{\ell r}$, the unstable periodic orbits $O_{\mathcal{R} \mathcal{L}^{n}}$ reach arbitrarily high periods. 


\subsection{Decreasing slope smaller than $\mathbf{- 1}$}

If the absolute value of the decreasing slope is raised above one (case 15 in Fig. 2), the resulting organizing center is considerably more complicated than the previous case. Note that a system very similar to this case was investigated in [8].

A characterizing feature of this case is the interaction between the so-called "channel" and "swirl" mechanisms. Both mechanisms affect an interval which has the partition boundary $x=0$ as one of its endpoints. On a channel-interval the system function has a positive slope. Each point in a channel is either mapped closer to the partition boundary, or to the other side of it. On a swirl-interval, the interval includes an unstable fixed point and the system function has a negative slope. Each point in a swirl is mapped to the other side of the fixed point and away from it, so that repeated iteration leads to the impression of the points swirling around the fixed point. Consequently, this means that in the current configuration of the slopes, $\mu_{\ell}>0$ implies that there exists a channel on the left side, and $\mu_{r}>0$ leads to a swirl on the right side.

The description below follows the bifurcation diagrams in Figs. $8-10$ from the left to the right. The first quadrant is the most complicated. In this quadrant we can identify four characteristic cases, depending on the location of the critical point $f_{\ell}(0)=\mu_{\ell}$ and the image $f_{\ell}\left(f_{r}\left(f_{r}(0)\right)\right)$ of the critical point $f_{r}(0)=\mu_{r}$ in relation to the fixed point $x^{\mathcal{R}}$ and $\mu_{r}$. Note that we have in any case $x^{\mathcal{R}}<\mu_{r}$, as the function $f_{r}$ is decreasing. This yields four parameter intervals, defined by the following conditions:

(i) $0<\mu_{\ell}<x^{\mathcal{R}}$

(ii) $f_{\ell}\left(f_{r}\left(f_{r}(0)\right)\right)<x^{\mathcal{R}}<\mu_{\ell}<\mu_{r}$

(iii) $x^{\mathcal{R}}<f_{\ell}\left(f_{r}\left(f_{r}(0)\right)\right)<\mu_{\ell}<\mu_{r}$

(iv) $\mu_{r}<\mu_{\ell}$.

In the first three cases the map has an absorbing interval given by $\left[f_{r}\left(f_{r}(0)\right), f_{r}(0)\right]$, and in the last case the absorbing interval is $\left[f_{r}\left(f_{\ell}(0)\right), f_{\ell}(0)\right]$. In Figs. $8-10$, the cases (i) to (iv) are marked with $\mathcal{I}_{1}$ to $\mathcal{I}_{4}$, respectively.

The transition from case (i) to case (ii) occurs when $\mu_{\ell}=x^{\mathcal{R}}$ (that means, at a homoclinic bifurcation of the fixed point $x^{\mathcal{R}}$ ), the transition from (ii) to (iii) occurs when $f_{\ell}\left(f_{r}\left(f_{r}(0)\right)\right)=x^{\mathcal{R}}$ (that means, at another homoclinic bifurcation of the fixed point $x^{\mathcal{R}}$ ). The transition from (iii) to (iv) occurs when $\mu_{\ell}=\mu_{r}$. For a description of the homoclinic bifurcations of the fixed point $x^{\mathcal{R}}$ occurring at the boundaries of this interval we refer to [22].

(i): In the parameter interval given by $0<\mu_{\ell}<x^{\mathcal{R}}$ (marked with $\mathcal{I}_{1}$ in Figs. $8-10$ ), there is a region of period adding. The basic orbits of this period adding scenario correspond to the symbolic sequences $O_{\mathcal{L} \mathcal{R}^{2 n}}$, $n \geq 1$. This is an effect of the swirl-mechanism. Points which are mapped into the right partition swirl $n$-times (with each rotation in the swirl taking up two iterations) around the unstable fixed point, until they are finally mapped back to the left partition. There is also another sequence of periodic orbits, which exist to the left of $O_{\mathcal{L R}^{2}}$ and which correspond to sequences of the form $O_{\mathcal{L}^{n}} \mathcal{R}^{2}$. This is an effect of the channel mechanism, which leads to an increasing number of points on the left side. The points on the right side rotate one time around the unstable fixed point and are then mapped back to the left side.

At first glance, the border of stability of this period adding seems to be harder to determine than in the increasing/increasing case since the system function is not invertible on the absorbing interval $\left[f_{r}\left(f_{r}(0)\right), f_{r}(0)\right]$. However, note that all sequences corresponding to stable orbits in this period adding have an even number of $\mathcal{R}$. We can take advantage of that by using the iterated function $f_{r r}(x)=f_{r} \circ f_{r}(x)$. We can use this function for the right side of the system here, because of the swirl described above. Whenever a point is mapped into the right side from the left, it arrives below the fixed point $x^{\mathcal{R}}$ and spends $2 n$ iterations on the right side, before it is mapped back to the left side, for some $n>1$. This means, we can consider a map with the left branch $f_{\ell}(x)$ and the right branch $f_{r r}(x)$, which reflects - under the given conditions - the behavior of the original map. The composite map $f_{\ell} / f_{r r}$ has two increasing slopes, with one slope larger than one, and it behaves exactly as it is described in $§ 3.2$. There is an absorbing interval $\left[f_{r r}(0), f_{\ell}(0)\right]$ and the composite map $f_{\ell} / f_{r r}$ is invertible as long as $f_{\ell}\left(f_{r r}(0)\right)>f_{r r}\left(f_{\ell}(0)\right)$. This means that the composite map becomes not invertible on its absorbing interval when $f_{\ell}\left(f_{r r}(0)\right)=f_{r r}\left(f_{\ell}(0)\right)$. In terms of system (1) this translates to the condition 
$f_{\ell}\left(f_{r}\left(f_{r}(0)\right)\right)=f_{r}\left(f_{r}\left(f_{\ell}(0)\right)\right)$, which leads after some algebraic transformations to an expression equal to the one of the curve $\tau$ (see Eq. 5), as it was already observed in [23].

On the other side of $\tau$, only chaotic dynamics occur. A region of bandcount adding follows. The end of the bandcount adding region is given by a crisis bifurcation (caused by a homoclinic bifurcation of the fixed point $x^{\mathcal{R}}$ mentioned above).

(ii): For the parameter interval defined by the condition $f_{\ell}\left(f_{r}\left(f_{r}(0)\right)\right)<x^{\mathcal{R}}<\mu_{\ell}<\mu_{r}$ (marked with $\mathcal{I}_{2}$ in Figs. 8 -10) we are not stating anything. In our experiments we observed only one-band attractors in this interval, but we can not exclude the possibility of multi-band attractors there.

(iii): In the parameter interval given by $x^{\mathcal{R}}<f_{\ell}\left(f_{r}\left(f_{r}(0)\right)\right)<\mu_{\ell}<\mu_{r}$ (marked with $\mathcal{I}_{3}$ in Figs. $8-10$ ) the behavior of the map can be described using a composite map slightly different than in the previous case. As the left boundary of the absorbing interval is given here by $f_{\ell}\left(f_{r}\left(f_{r}(0)\right)\right)$ and the function $f_{\ell}$ is increasing, the condition $f_{\ell}\left(f_{r}\left(f_{r}(0)\right)\right)>x^{\mathcal{R}}$ guarantees that all points $x<0$ in the absorbing interval are mapped to the right side above the fixed point $x^{\mathcal{R}}$, in one step. This means that all points which are mapped to the right side from the left side take $2 n+1$ steps rotating around the fixed point $x^{\mathcal{R}}$ for $n \geq 1$. Therefore, in this parameter interval $x^{\mathcal{R}}<f_{\ell}\left(f_{r}\left(f_{r}(0)\right)\right)<\mu_{\ell}<\mu_{r}$, periodic orbits corresponding to the symbolic sequences $O_{\mathcal{L R}^{2 n+1}}, n \geq 1$ exist. The symbolic sequences of these orbits consist of one $\mathcal{L}$ and an odd number of $\mathcal{R}$ s, or, in other words, are starting with $\mathcal{L} \mathcal{R}$ which is followed by an even number of $\mathcal{R} \mathrm{s}$. This behavior can be described by considering the composite map with the left branch $f_{\ell r}(x)=f_{r} \circ f_{\ell}(x)$ and the right branch $f_{r r}(x)$. It can easily be seen that under the given conditions the composite map $f_{\ell r} / f_{r r}$ reflects the behavior of the original map and that it has a decreasing slope on the left and an increasing slope on the right.

The increasing slope of $f_{r r}$ is always larger than 1 , but the slope of $f_{\ell r}$ can be smaller or larger than -1 . Both cases lead to different behaviors. Let us consider first the case that the slope of $f_{\ell r}$ is larger than -1 (this case is shown in Fig. 8). In this case, the interval begins with the bandcount incrementing scenario, which ends in a stability border. After the stability border a region of period incrementing appears, which consists of the orbits mentioned above, corresponding to the symbolic sequences $O_{\mathcal{L R}^{2 n+1}}, n \geq 1$. The explanation for that follows directly from the composite map $f_{\ell r} / f_{r r}$. The composite map $f_{\ell r} / f_{r r}$ has a decreasing slope larger than -1 and an increasing slope larger than one, and it behaves as described in $\S 4.2$ (however, note that the composite map $f_{\ell r} / f_{r r}$ is the conjugate case to the one described in $\S 4.2$ - decreasing/increasing instead of increasing/decreasing). Therefore, the composite map $f_{\ell r} / f_{r r}$ shows an incrementing cascade formed by the orbits from the family $O_{\mathcal{L R}^{n}}, n \geq 1$, and in the original map this cascade corresponds to the incrementing cascade formed by the orbits $O_{\mathcal{L R}^{2 n+1}}, n \geq 1$. These orbits are stable up to some $n$ given by the the largest number which satisfies the condition $\left|a_{\ell} a_{r}^{2 n+1}\right|<1$ (in Fig. 8, n=7) and unstable thereafter. Hence, the border collision bifurcation of the stable orbit with the highest period represents also the stability boundary separating the period incrementing scenario from the bandcount incrementing one (in Fig. 8 this border collision bifurcation is marked with $\left.\xi_{\mathcal{L R}^{15}}\right)$.

If the slope of $f_{\ell r}$ is smaller than -1 (see Figs. 9 and 10), the situation is conjugate to the case considered in $§ 4.4$. In this case, no stable orbits are possible and we have only the bandcount incrementing scenario.

(iv): The rest of the first quadrant and the second quadrant are covered by the parameter interval given by $\mu_{r}<\mu_{\ell}$ (marked with $\mathcal{I}_{4}$ in Figs. $8-10$ ). It contains another instance of the period incrementing scenario, formed by the orbits with the symbolic sequences $O_{\mathcal{R} \mathcal{L}^{n}}$, as proved in [13]. As one can immediately see, by contrast to the cases (i) and (iii), in the case (iv) the mechanism leading to the appearance of all orbits $O_{\mathcal{R}} \mathcal{L}^{n}$ is not the swirl but a channel on the left side, which exists because of $0<a_{\ell}<1$ and $\mu_{\ell}>0$. However, not all these orbits are stable. The stable orbit with the smallest period can be determined with the condition $\left|a_{r} a_{\ell}^{n}\right|<1$. All periodic orbits corresponding to values of $n$ larger than that will be stable. In Fig. $9, n=2$ and the border collision is marked with $\xi_{\mathcal{L} \mathcal{R} \mathcal{L}}$. In Fig. 10, $n=27$ and the border collision is marked with $\xi_{\mathcal{L} \mathcal{R} \mathcal{L}^{26}}$. This period incrementing can also be seen in Fig. 8 near $\phi=\pi$, though it is very small and compressed in this figure. $\mathrm{X}$ 


\subsection{Both slopes with an absolute value larger than 1}

If both slopes have an absolute value larger than 1 (case 16 in Fig. 2) in the increasing/decreasing case there is either chaos or divergence. The description below follows the bifurcation diagram in Fig. 11 from the left to the right.

On the left, in the interval defined by the condition $0<\mu_{\ell}<x^{\mathcal{R}}$ (that means, by the same condition as the case (i) in the previous section), there is a region organized by the bandcount adding scenario. In this region, the system function is not invertible on the absorbing interval which in this case is given by $\left[f_{r}\left(f_{r}(0)\right), f_{r}(0)\right]$

(recall that $\mu_{\ell}<x^{\mathcal{R}}$ implies also $\mu_{\ell}<\mu_{r}$ ). On the left there is divergence resulting from the boundary crisis caused by the collision of the fixed point $x^{\mathcal{L}}$ with the boundary of the attractor, which can be determined by

$$
f_{r}\left(f_{r}(0)\right)=x^{\mathcal{L}} \Rightarrow \eta_{r r}:=\left\{\left(a_{\ell}, a_{r}, \mu_{\ell}, \mu_{r}\right) \mid \frac{\mu_{\ell}}{\mu_{r}}=-a_{\ell} a_{r}+a_{r}-a_{\ell}+1\right\}
$$

After the bandcount adding region follows a one-band attractor, followed by a bandcount incrementing region. In this region of bandcount incrementing, the absorbing interval changes to $\left[f_{r}\left(f_{\ell}(0)\right), f_{\ell}(0)\right]$. Note that depending on the particular values of $a_{\ell}$ and $a_{r}$ the bandcount incrementing scenario (as well as its substructures) may be present or not.

After the region of bandcount incrementing follows divergence. The border to divergence is given by the collision of the fixed point $x^{\mathcal{L}}$ with the boundary of the attractor in a boundary crisis, which can be determined by Eq. (8). 

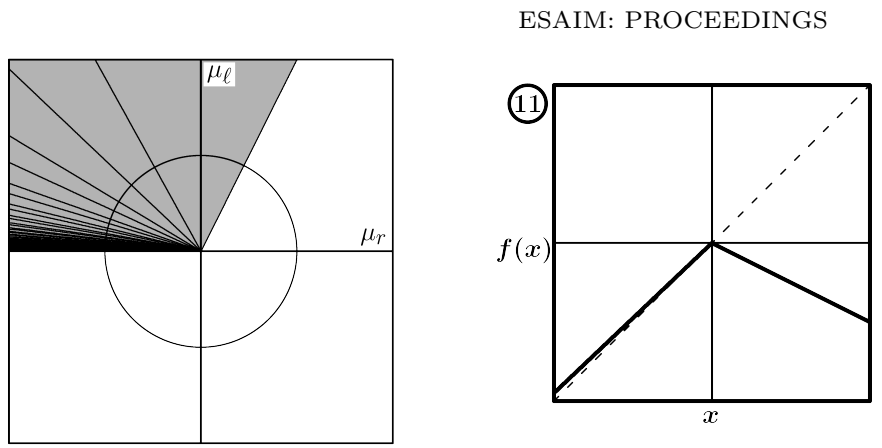

(B)

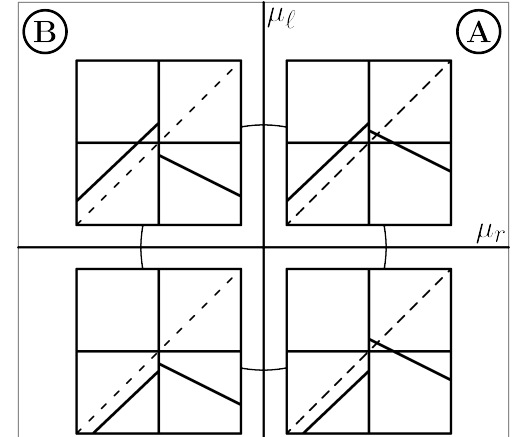

(C)
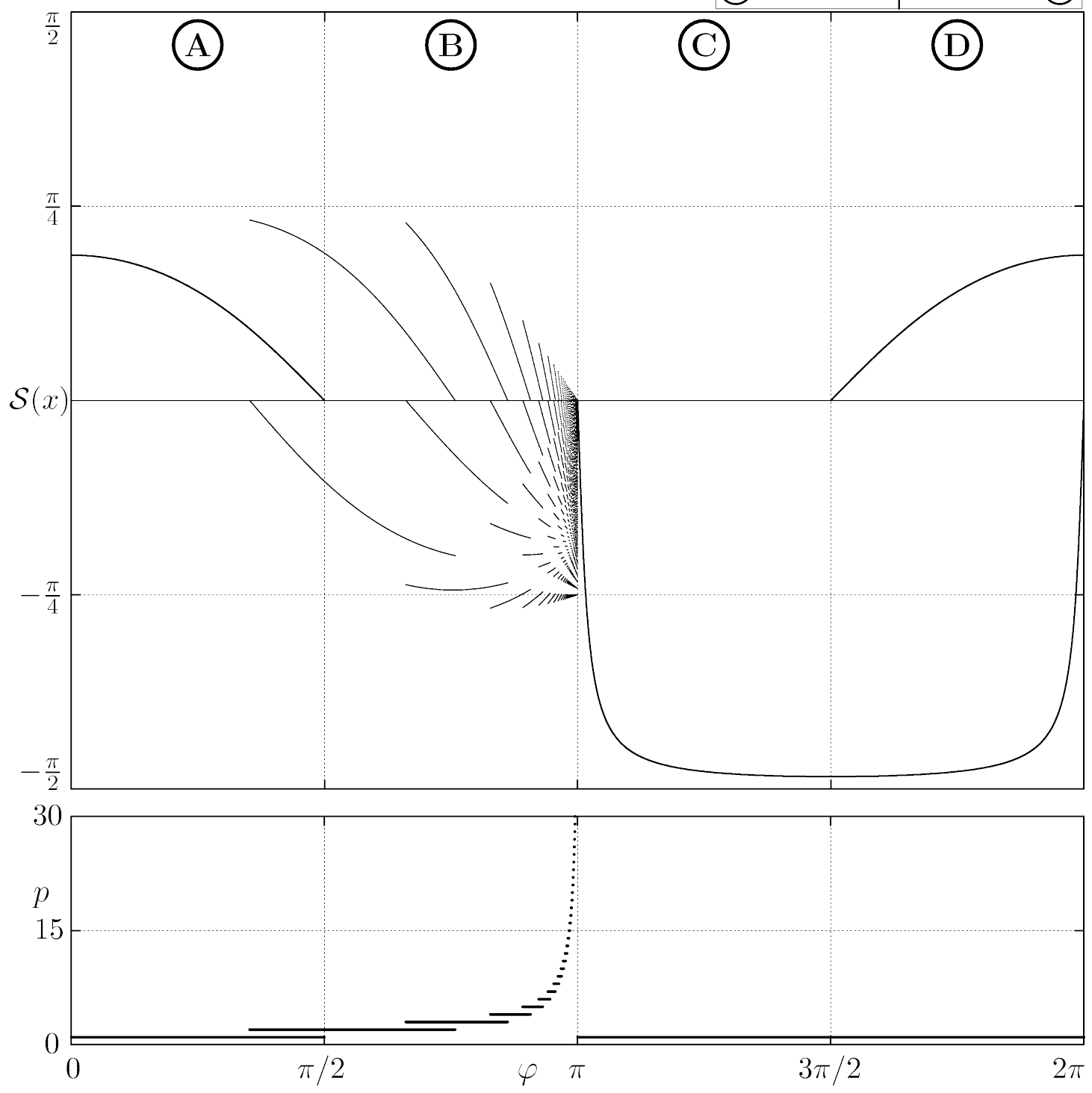

Figure 6. Increasing / decreasing case with $0<a_{\mathcal{L}}<1,-1<a_{\mathcal{R}}<0$.

The second quadrant is covered by the period incrementing scenario, in the remaining three quadrants the fixed points are stable (in the fourth quadrant they coexist, in the first quadrant the existence region of $x^{\mathcal{R}}$ overlaps with the existence region of the 2 -cycle). $\left(a_{\mathcal{L}}=0.95, a_{\mathcal{R}}=-0.5\right)$ 
(B)
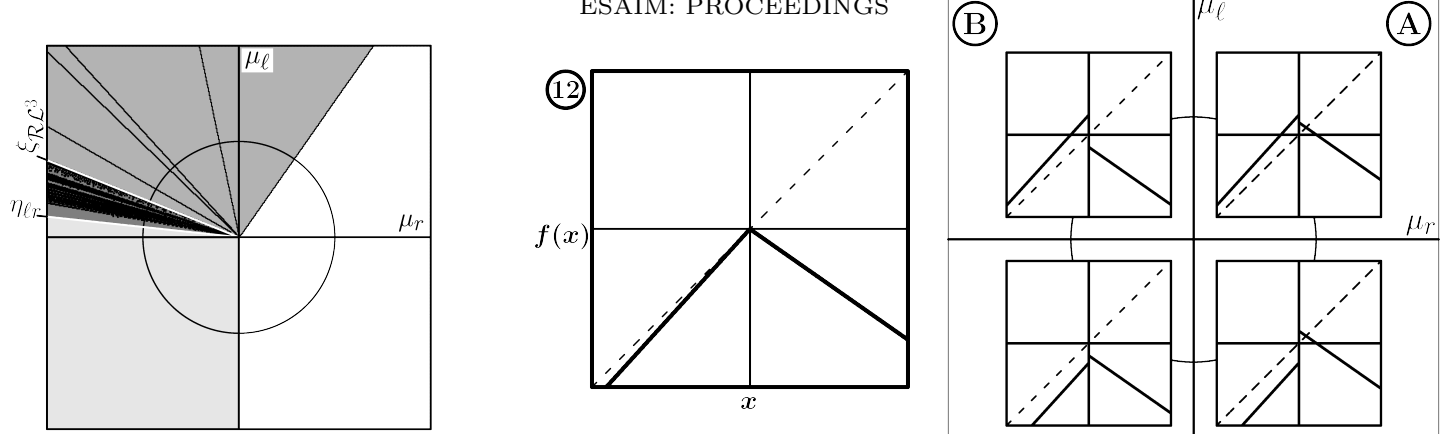

(C)
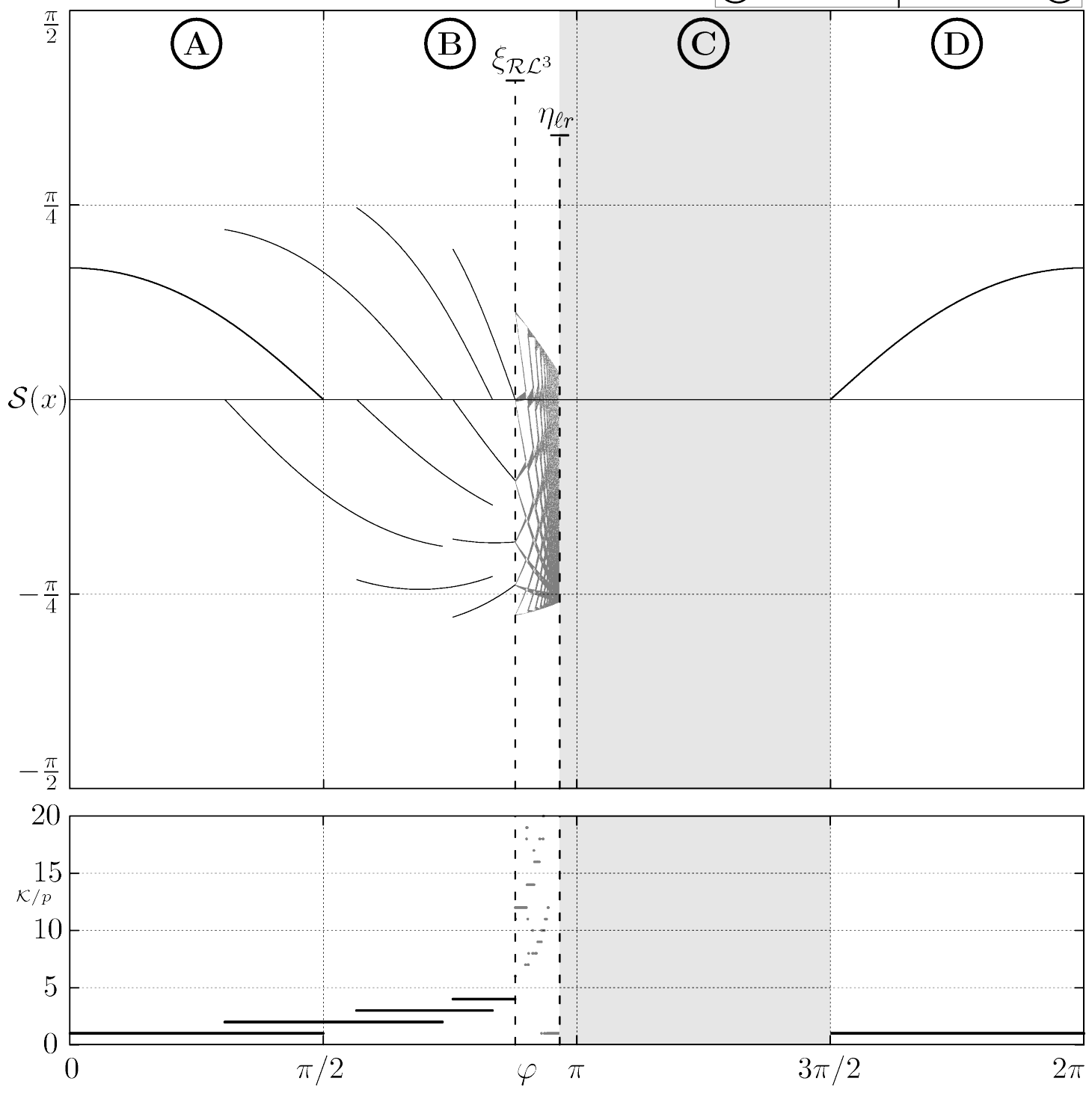

FiguRe 7. Increasing / decreasing case with $a_{\mathcal{L}}>1,-1<a_{\mathcal{R}}<0$.

In the second quadrant exist period incrementing, chaotic attractors organized by bandcount incrementing and divergence. In the remaining quadrants there is divergence and the existence regions of the stable fixed point $x^{\mathcal{R}}$ and of the 2-cycle. $\left(a_{\mathcal{L}}=1.1, a_{\mathcal{R}}=-0.7\right)$ 

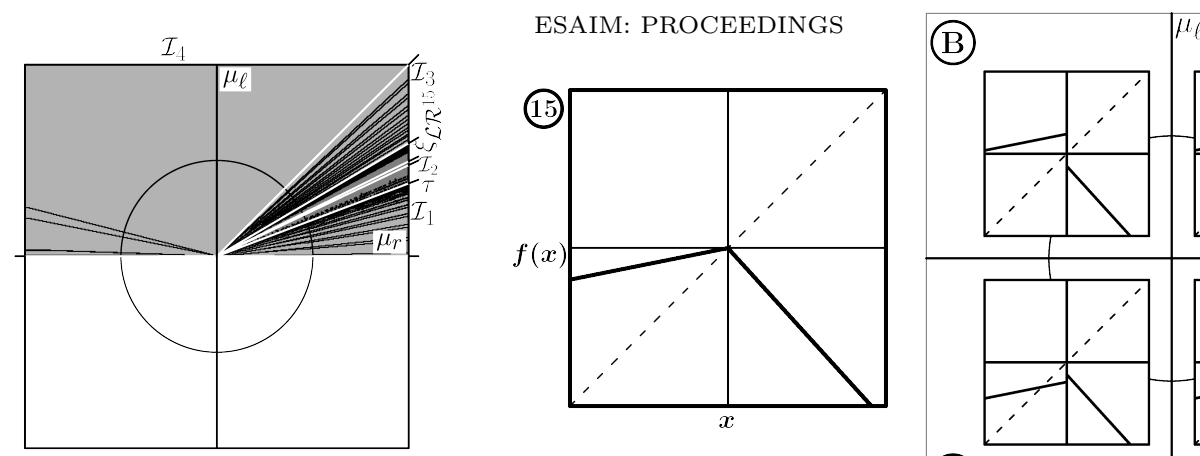

(C)
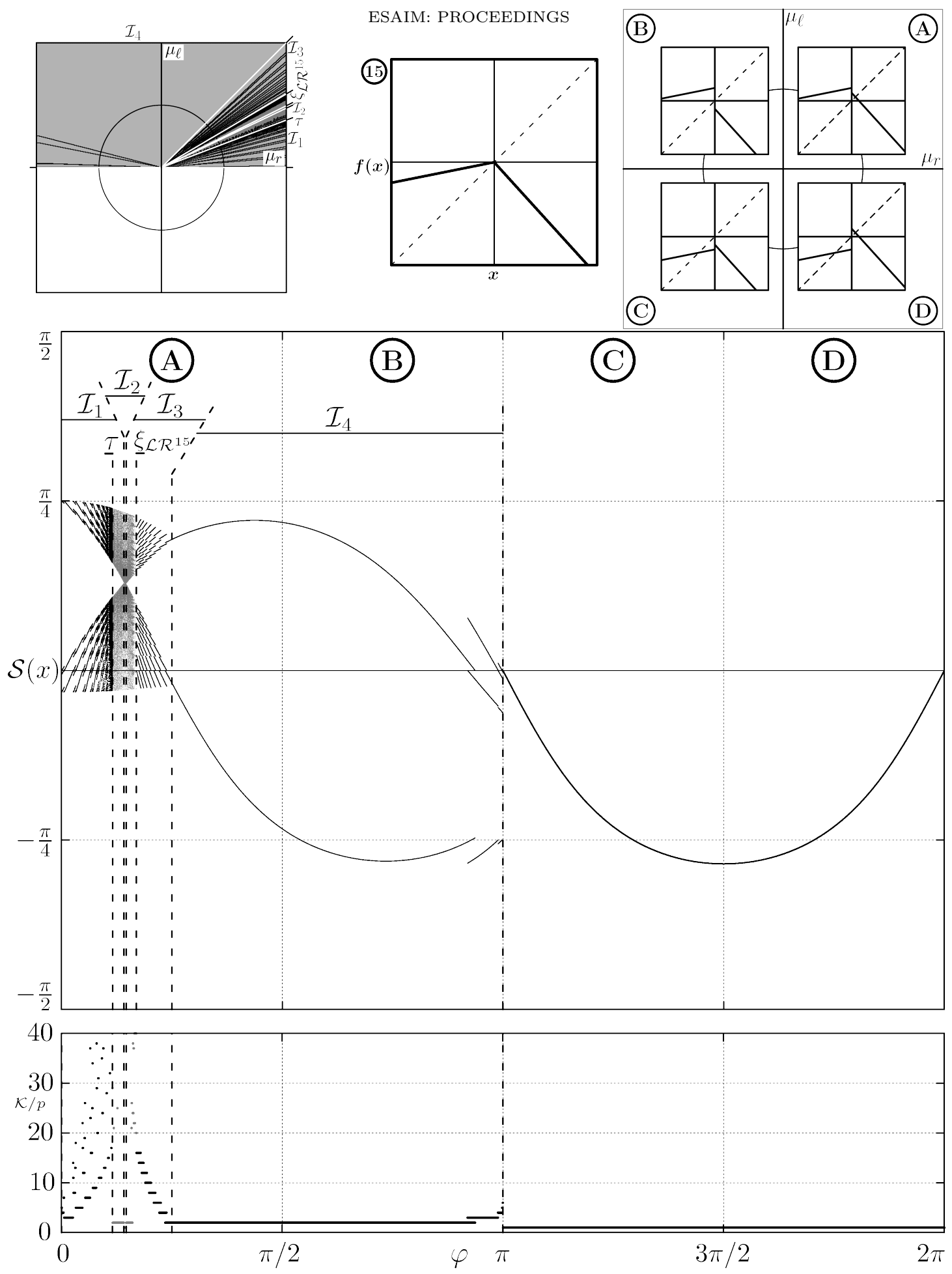

FiguRE 8. Increasing / decreasing case with $0<a_{\mathcal{L}}<1, a_{\mathcal{R}}<-1$.

In the first quadrant exist period adding and period incrementing, separated by chaotic attractors. In the second quadrant exists the 2-cycle and period incrementing. Third and fourth quadrants contain the stable fixed point $x^{\mathcal{R}}$. $\left(a_{\mathcal{L}}=0.2, a_{\mathcal{R}}=-1.1\right)$ 

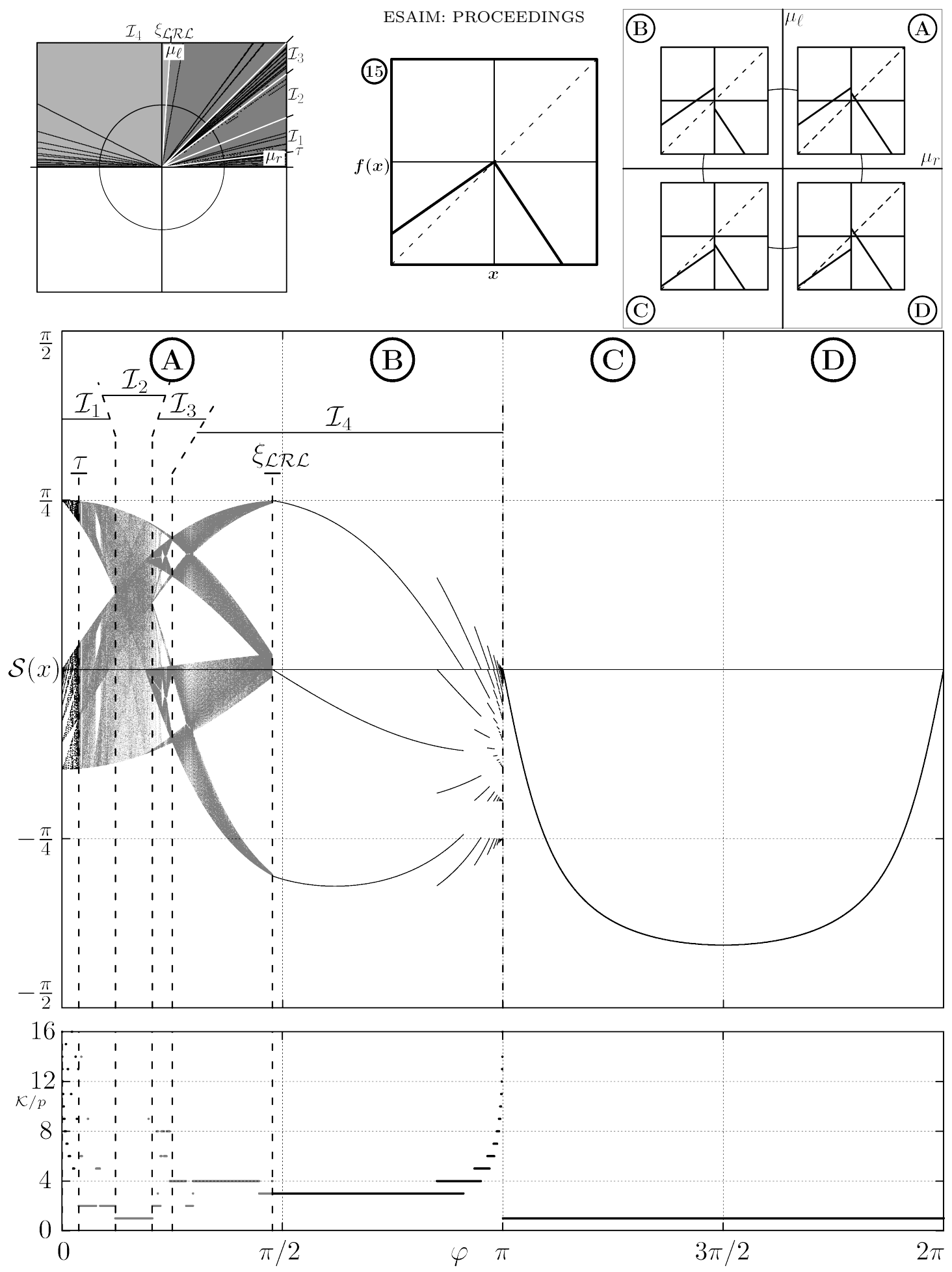

FiguRE 9. Increasing / decreasing case with $0<a_{\mathcal{L}}<1, a_{\mathcal{R}}<-1$.

In the first quadrant exist period adding and chaotic attractors, which are organized by bandcount adding and incrementing. The second quadrant is covered by period incrementing. Third and fourth quadrants contain $x^{\mathcal{R}} . \quad\left(a_{\mathcal{L}}=0.7\right.$, $\left.a_{\mathcal{R}}=-1.5\right)$ 


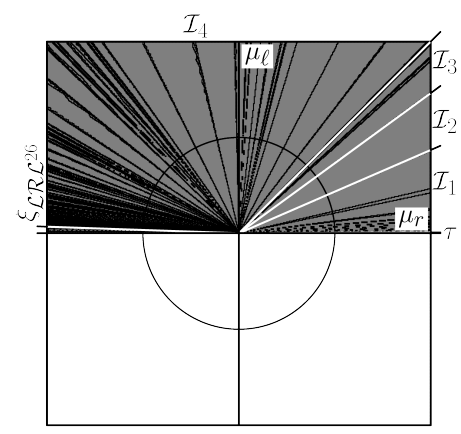

(B)
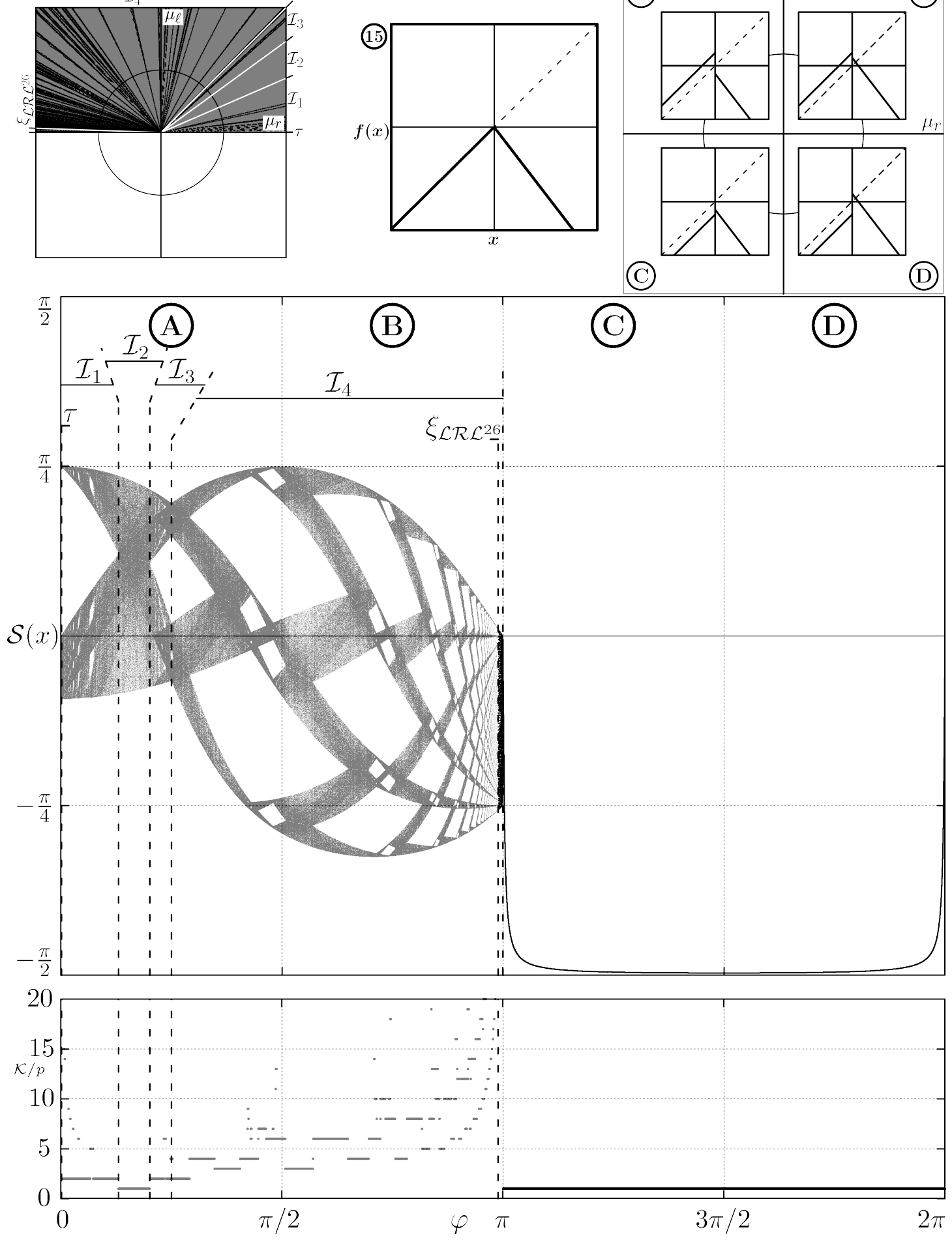

FIGURE 10. Increasing / decreasing case with $0<a_{\mathcal{L}}<1, a_{\mathcal{R}}<-1$.

In the first quadrant exist period adding (for $\phi \in(0, \tau)$ ) and chaotic attractors organized by bandcount adding and incrementing scenarios. The second quadrant contains bandcount incrementing, with period incrementing at the right end. Third and fourth quadrants contain $x^{\mathcal{R}} .\left(a_{\mathcal{L}}=0.99, a_{\mathcal{R}}=-1.3\right)$ 
(B)
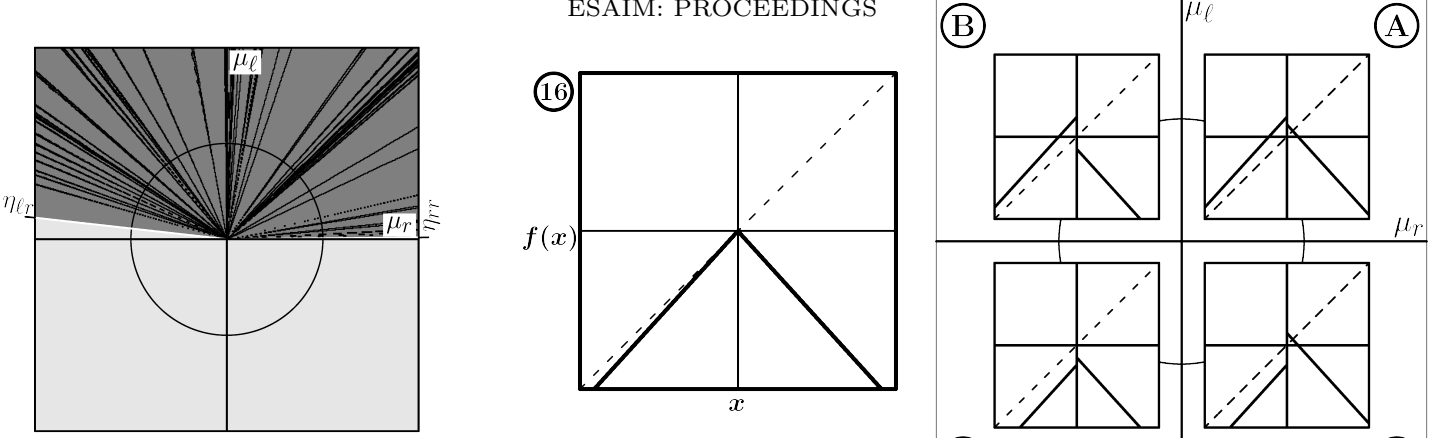

(A)

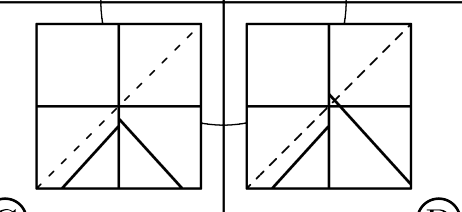

(C)

(D)
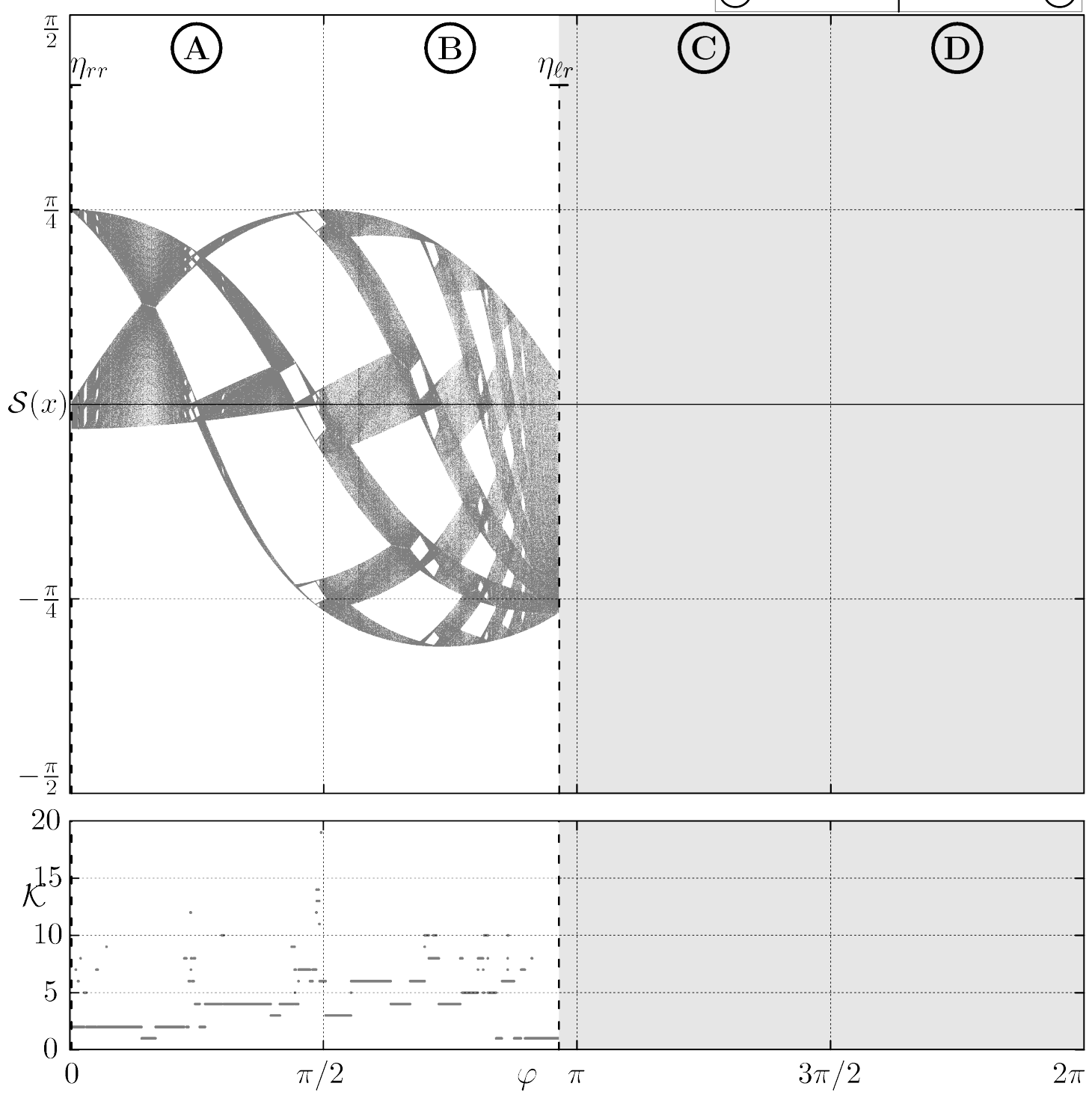

FiguRE 11. Increasing / decreasing case $a_{\mathcal{L}}>1, a_{\mathcal{R}}<-1$.

In the first and second quadrants exist chaotic attractors organized by bandcount adding and incrementing scenarios. The second, third and fourth quadrants (and a small part of the first one) contain the region of divergence. $\left(a_{\mathcal{L}}=1.1, a_{\mathcal{R}}=-1.1\right)$. 


\section{Decreasing/Decreasing CASe}

\subsection{No slope smaller than -1}

No slope larger than one in the decreasing/decreasing case (case 10 in Fig. 2) leads to the most simple organizing center in piecewise linear maps. The bifurcation scenario consists of the stable fixed points and a stable period 2 orbit and nothing else. This case is depicted in Fig. 12.

\subsection{One slope smaller than -1}

If the absolute value of one slope is increased above one (case 14 in Fig. 2), there may exist the period adding scenario, the period incrementing scenario and regions of chaos and divergence. The arrangement of these regions is similar to the increasing/decreasing case with the decreasing slope larger than one (see [8] for a comparison of both cases). The description below follows the bifurcation diagram in Fig. 13 from the left to the right. Only the first quadrant shows non trivial bifurcation scenarios. It can be subdivided into four parts, depending on the location of the critical point $f_{r}(0)=\mu_{r}$ and it's image $f_{\ell}\left(f_{r}\left(f_{r}(0)\right)\right)$ with respect to the fixed point $x^{\mathcal{R}}$. This yields four parameter intervals, defined the following conditions:
(i) $0<\mu_{\ell}$ and $f_{\ell}\left(f_{r}\left(f_{r}(0)\right)\right)<x^{\mathcal{R}}$
(ii) $0<\mu_{\ell}<x^{\mathcal{R}}<f_{\ell}\left(f_{r}\left(f_{r}(0)\right)\right)<\mu_{r}$
(iii) $0<f_{\ell}\left(f_{r}\left(f_{r}(0)\right)\right)<\mu_{r}$ and $x^{\mathcal{R}}<\mu_{\ell}$
(iv) $\mu_{r}<f_{\ell}\left(f_{r}\left(f_{r}(0)\right)\right)$.

In Fig. 13 the cases (i) to (iv) are marked with $\mathcal{I}_{1}$ to $\mathcal{I}_{4}$, respectively. In Fig. 14 only the cases (ii)-(iv) are marked, since case (i) does not exist for the values of the slopes corresponding to that figure. Note also that the conditions (i)-(iii) imply $\mu_{\ell}<\mu_{r}$, since the function $f_{r}$ is decreasing here, and $\mu_{\ell}<f_{\ell}\left(f_{r}\left(f_{r}(0)\right)\right.$ ) (this condition is always true here as the function $f_{\ell}$ is decreasing). Therefore, in these cases the map has an absorbing interval given by $\left[f_{r}\left(f_{r}(0)\right), f_{r}(0)\right]$. Recall also that we have in any case $x^{\mathcal{R}}<\mu_{r}$, as the function $f_{r}$ is decreasing.

The transition from case (i) to case (ii) occurs when $f_{\ell}\left(f_{r}\left(f_{r}(0)\right)\right)=x^{\mathcal{R}}$. The transition from case (ii) to case (iii) occurs when $\mu_{\ell}=x^{\mathcal{R}}$. Both transitions represent homoclinic bifurcation of the fixed point $x^{\mathcal{R}}$ which is homoclinic in the interval (ii). The transition from case (iii) to case (iv) occurs when $f_{\ell}\left(f_{r}\left(f_{r}(0)\right)\right)=\mu_{r}$.

(i): In the parameter interval given by the conditions ${ }^{4} 0<\mu_{\ell}$ and $f_{\ell}\left(f_{r}\left(f_{r}(0)\right)\right)<x^{\mathcal{R}}$ (marked with $\mathcal{I}_{1}$ in Fig. 13) the period incrementing scenario can be observed. The orbits forming period incrementing scenario correspond to the symbolic sequences $O_{\mathcal{L R}^{2 n}}$. As in the increasing/decreasing case with the decreasing slope larger than one, the scenario can be explained with the help of a case already discussed before. Note that each symbolic sequence in this period incrementing scenario starts with a single letter $\mathcal{L}$ (as for all $x<0$ we have $f(x)>0$ ) and has an even number of $\mathcal{R} \mathrm{s}$ (due to the fact that periodic orbits enter the swirl on the right side below the fixed point). Therefore, we can consider the composite map which uses $f_{\ell}(x)$ as the left branch, and $f_{r r}(x)$ as the right branch, which reflects - under the given conditions - the behavior of the original map.

The resulting composite map $f_{\ell} / f_{r r}$ corresponds to the decreasing/increasing case, with the increasing slope larger than one, and it is conjugate to the case described in $\S 4.2$. Therefore, the composite map $f_{\ell} / f_{r r}$ shows an incrementing cascade formed by the orbits from the family $O_{\mathcal{L R}^{n}}, n \geq 1$, which corresponds, in the original map, to the incrementing cascade formed by the orbits $O_{\mathcal{L R}^{2 n}}, n \geq 1$. Not all orbits of the period incrementing scenario are stable. The stable periodic orbit with the highest period is the orbit which satisfies $\left|a_{\ell} a_{r}^{2 n}\right|<1$ with the largest $n$ (in Fig. 13, $n=8$ ). The border collision bifurcation at which this orbit disappears is the stability border of the period incrementing scenario (in Fig. 13, it is marked with $\xi_{\mathcal{L R}^{16}}$ ). In the chaotic domain, after the period incrementing scenario follows the bandcount incrementing scenario. At the end of the bandcount incrementing scenario, the absorbing interval $\left[f_{r r}(0), f_{\ell}\left(f_{r r}(0)\right)\right]$ of the composite map $f_{\ell} / f_{r r}$ collides with the fixed point $x^{\mathcal{R}}$ (this is the homoclinic bifurcation of $x^{\mathcal{R}}$ mentioned above).

\footnotetext{
${ }^{4}$ If both conditions can not be fulfilled simultaneously for a given combination of the slopes $a_{\ell}, a_{r}$, then this case does not appear.
} 
(ii): Similar to the case discussed in $\S 4.3$, we exclude from our considerations the parameter interval $0<$ $\mu_{\ell}<x^{\mathcal{R}}<f_{\ell}\left(f_{r}\left(f_{r}(0)\right)\right)<\mu_{r}$ (marked with $\mathcal{I}_{2}$ in Figs. 13 and 14). For this parameter interval, we observed one-band attractors only, but there is still no proof that multi-band attractors can not exist in this case.

(iii): In the parameter interval given by the conditions ${ }^{4} x^{\mathcal{R}}<\mu_{\ell}$ and $f_{\ell}\left(f_{r}\left(f_{r}(0)\right)\right)<\mu_{r}$ (marked with $\mathcal{I}_{3}$ in Figs. 13 and 14) periodic orbits corresponding to the symbolic sequences $O_{\mathcal{L R}^{2 n+1}}$ exist, as well as periodic orbits that can be derived from $O_{\mathcal{L R}^{2 n+1}}$ using the symbolic sequences adding scheme. The behavior of the map can be explained using the composite map with $f_{\ell r}$ as the left branch and $f_{r r}$ as the right branch. The explanation why under the given conditions this composite map reflects the behavior of the original map is similar to the case (i) above, with the only difference that a periodic orbit is mapped from the left side to the right one above the unstable fixed point. As a consequence, the number of $\mathcal{R} \mathrm{s}$ in the resulting symbolic sequences is not even but odd. The slope corresponding to $f_{r r}$ is always larger than one, but the slope corresponding to $f_{\ell r}$ can be smaller or larger than one. Let us consider first what happens if the slope of $f_{\ell r}$ is smaller than one. In this case, the interval begins with the bandcount adding scenario, followed by period adding. The basic orbits of this adding scenario correspond to the sequences $O_{\mathcal{L R}^{2 n+1}}$ mentioned above. In this adding scenario the system function is not invertible on the absorbing interval. However, the composite map $f_{\ell r} / f_{r r}$ is in the increasing/increasing configuration with one slope larger than one and it behaves as described in $\S 3.2$. This means that there is an absorbing interval $\left[f_{r r}(0), f_{\ell r}(0)\right]$ on which the system is invertible as long as $f_{\ell r}\left(f_{r r}(0)\right)>f_{r r}\left(f_{\ell r}(0)\right)$. Consequently, the stability border between bandcount adding and period adding can be determined by $f_{\ell r}\left(f_{r r}(0)\right)=f_{r r}\left(f_{\ell r}(0)\right)$, which translates to the condition $f_{r}\left(f_{\ell}\left(f_{r}\left(f_{r}(0)\right)\right)\right)=f_{r}\left(f_{r}\left(f_{r}\left(f_{\ell}(0)\right)\right)\right)$ in system (1), and which leads after some algebraic transformations to an expression equal to the one of the curve $\tau$ (see Eq. 5).

If the slope of $f_{\ell r}$ is larger than 1 , the interval may contain the bandcount adding scenario (which may be present or not, depending on $\left.a_{\ell}, a_{r}\right)$.

(iv): Depending on the slopes $a_{\ell}, a_{r}$, in the parameter interval given by the condition $f_{\ell}\left(f_{r}\left(f_{r}(0)\right)\right)>\mu_{r}$ (marked with $\mathcal{I}_{4}$ in Figs. 13 and 14) either a stable 2-periodic orbit $O_{\mathcal{L R}}$ appears (if $a_{\ell} a_{r}<1$ ), or divergence (otherwise).

Note that when the 2-periodic orbit $O_{\mathcal{L R}}$ is unstable, all other orbits $O_{\mathcal{L R}^{n}}$ with $n>1$ are unstable as well. Therefore, in the cases (i) and (iii) no period incrementing and no period adding scenarios are possible, but only chaos. The boundary between case (iii) and case (iv) can be determined as

$$
f_{\ell}\left(f_{r}\left(f_{r}(0)\right)\right)=\mu_{r} \quad \Rightarrow \quad \eta_{r}^{\mathcal{R} \mathcal{L}}:=\left\{\left(a_{\ell}, a_{r}, \mu_{\ell}, \mu_{r}\right) \mid \frac{\mu_{\ell}}{\mu_{r}}=1-a_{r} a_{\ell}-a_{\ell}\right\}
$$

This corresponds to the boundary crisis caused by the collision of the right point $x^{\mathcal{R L}}$ of the unstable orbit $O_{\mathcal{L R}}$ with the boundary of the attractor.

In both cases (when $O_{\mathcal{L R}}$ is stable or not), on the right side of the bifurcation diagram shown in Figs. 13 and 14 (that means, for $\mu_{\ell}<0$ ) there exists the stable fixed point $x^{\mathcal{L}}$, which is however not necessarily globally attracting (see [24]).

\subsection{Both slopes smaller than -1}

If both slopes are smaller than -1 (case 13 in Fig. 2) all periodic orbits are unstable and the only bounded attractors are chaotic. Note that in this case divergence is possible for any combination of slopes and offsets. The bifurcation structure inside the domain of chaotic attractors seems to be complicated in this case (Fig. 15), can however be explained easily in the following way. Consider first the function $f_{r}$. For $\mu_{r}>0$ it has a fixed point, which is unstable due to $a_{r}<-1$. As we have already seen in $\S 5.2$, the appearance of the periodic orbits in this case is based on the swirl-mechanism: when $\mu_{\ell}>0$ (and hence every point $x<0$ is immediately mapped to the right side) the rotation of the orbits around the unstable fixed point $x^{\mathcal{R}}$ causes the complete family of basic orbits $O_{\mathcal{L R}^{n}}$ for all $n$ to emerge. Moreover, depending on the fact whether the orbit enters the swirl below or above the fixed point $x^{\mathcal{R}}$, it has an even or an odd number of $R$ s. Therefore, for $0<\mu_{\ell}<x^{\mathcal{R}}$ we observe a family of orbits $O_{\mathcal{L R}^{2 n}}$ with odd periods, and for $x^{\mathcal{R}}<\mu_{\ell}$ - the family $O_{\mathcal{L R}^{2 n+1}}$. As already mentioned, all 
these orbits are unstable. Note also that the situation regarding the function $f_{\ell}$ is similar: for $\mu_{\ell}<0$ there exists an unstable fixed point $x^{\mathcal{L}}$, and for $\mu_{r}<0$ all orbits are mapped from the right side to the left within one step. As a consequence, we obtain also in this case two families of unstable orbits, namely $O_{\mathcal{R} \mathcal{L}^{2 n}}$ and $O_{\mathcal{R} \mathcal{L}^{2 n+1}}$. All four families exist (in suitable intervals for $\mu_{\ell}, \mu_{r}$ ) for all $a_{\ell}, a_{r}$ and may be involved in two bifurcation scenarios in the chaotic domain, namely in the bandcount adding and in the bandcount incrementing scenario. However, it is worth to emphasize that depending on the particular values of $a_{\ell}$ and $a_{r}$ both scenarios (as well as their substructures) may appear or not.

The described situation is illustrated in Fig. 15. As one can see, in the presented case $a_{\ell}=a_{r}$ the bifurcation structure is symmetric with respect to the line $\mu_{\ell}=-\mu_{r}$ and the non-trivial behavior can be found in the third quadrant (marked with $\mathrm{C}$ ) and in the surrounding quadrants (marked with A and D in Fig. 15). Therefore, the description below follows the bifurcation diagram shown in Fig. 15 from the left to the right starting from the third quadrant and not from the first one. We can identify the following parameter intervals:

(i) $\mu_{\ell}<f_{r}\left(f_{\ell}\left(f_{\ell}(0)\right)\right)<0$ and $\mu_{r}<x^{\mathcal{L}}$

(ii) $\mu_{\ell}<f_{r}\left(f_{\ell}\left(f_{\ell}(0)\right)\right)<x^{\mathcal{L}}<\mu_{r}$

(iii) $x^{\mathcal{L}}<f_{r}\left(f_{\ell}\left(f_{\ell}(0)\right)\right)$ and $\mu_{\ell}, \mu_{r}<0$

(iv) $x^{\mathcal{R}}<f_{\ell}\left(f_{\ell}(0)\right)$ and $\mu_{\ell}<0$

(v) $f_{\ell}\left(f_{\ell}(0)\right)<x^{\mathcal{R}}, x^{\mathcal{L}}<f_{r}\left(f_{r}(0)\right)$ and $\mu_{\ell}<0, \mu_{r}>0$

(vi) $f_{r}\left(f_{r}(0)\right)<x^{\mathcal{L}}$ and $\mu_{r}>0$

(vii) $f_{\ell}\left(f_{r}\left(f_{r}(0)\right)\right)<x^{\mathcal{R}}$ and $\mu_{\ell}, \mu_{r}>0$

(viii) $\mu_{\ell}<x^{\mathcal{R}}<f_{\ell}\left(f_{r}\left(f_{r}(0)\right)\right)<\mu_{r}$

(ix) $0<f_{\ell}\left(f_{r}\left(f_{r}(0)\right)\right)<\mu_{r}$ and $x^{\mathcal{R}}<\mu_{\ell}$

The transitions from case (i) to case (ii), from case (ii) to case (iii) and from case (v) to case (vi) are associated with homoclinic bifurcations of the fixed point $x^{\mathcal{L}}$. Similarly, the transitions from case (iv) to case (v), from case (vii) to case (viii), and from case (viii) to case (ix) are associated with homoclinic bifurcations of the fixed point $x^{\mathcal{R}}$. The transitions from case (iii) to case (iv) and from case (vi) to case (vii) are given by the conditions $\mu_{r}=0$ and $\mu_{\ell}=0$, respectively, that means by the border collision bifurcations of the fixed points $x^{\mathcal{R}}$ and $x^{\mathcal{L}}$.

In the following we are not stating anything for the cases (ii), (iv), (vi) and (viii).

(i): In the interval $\mathcal{I}_{1}$, defined by the conditions $\mu_{\ell}<f_{r}\left(f_{\ell}\left(f_{\ell}(0)\right)\right)<0$ and $\mu_{r}<x^{\mathcal{L}}$, the chaotic region is structured by the bandcount adding scenario. In this interval, the basic periodic orbits with the sequences $O_{\mathcal{R} \mathcal{L}^{2 n+1}}, n \geq 1$ exist, as well as the orbits that can be derived from them using the symbolic sequences adding scheme. As mentioned above, a swirl exists on the left side, since $\mu_{\ell}<0$. Points on the right side are always mapped to the left side in one iteration, below the fixed point $x^{\mathcal{L}}$. From this follows that points spend $2 n+1$ steps, rotating around the swirl on the left side, before they are mapped back to the right side, for some $n \geq 1$. Therefore, we can consider a composite map in which we use $f_{\ell \ell}$ for values $x<0$ and $f_{r \ell}$ for $x>0$. This yields an increasing/increasing system with both slopes larger than one, as described in $\S 3.3$. There is an absorbing interval $\left[f_{r \ell}(0), f_{\ell \ell}(0)\right]$ on which the composite map is not invertible. This absorbing interval exists for parameter values in interval $\mathcal{I}_{1}$. The right boundary of the interval $\mathcal{I}_{1}$ is given by the condition $\mu_{r}=x^{\mathcal{L}}$ and is therefore equal to $\eta_{r}$ (see Eq. (7)). The left boundary of the interval $\mathcal{I}_{1}$ is given by

$$
\mu_{\ell}=f_{r}\left(f_{\ell}\left(f_{\ell}(0)\right)\right) \Rightarrow \eta_{\ell}^{\mathcal{R} \mathcal{L}}:=\left\{\left(a_{\ell}, a_{r}, \mu_{\ell}, \mu_{r}\right) \mid \frac{\mu_{\ell}}{\mu_{r}}=\frac{1}{1-a_{\ell} a_{r}-a_{r}}\right\}
$$

At the boundaries of interval $\mathcal{I}_{1}$, the absorbing interval collides with one of the fixed points of the composite map $f_{\ell \ell} / f_{r \ell}$. For the left boundary, this is the right fixed point, given by $f_{r \ell}(x)=x$, which is a point of the unstable periodic orbit $O_{\mathcal{L R}}$. At the right boundary of interval $\mathcal{I}_{1}$ the absorbing interval collides with the left fixed point of the composite map, given by $f_{\ell \ell}(x)=x$, which is equal to the fixed point $x^{\mathcal{L}}$ of the original map. 
(iii): In the interval $\mathcal{I}_{3}$, defined by the conditions $x^{\mathcal{L}}<f_{r}\left(f_{\ell}\left(f_{\ell}(0)\right)\right)$ and $\mu_{\ell}, \mu_{r}<0$ the chaotic region is structured by the bandcount incrementing scenario. In this interval, the basic periodic orbits with the sequences $O_{\mathcal{R} \mathcal{L}^{2 n}}, n \geq 1$ exist. As in interval $\mathcal{I}_{1}$, there is a swirl on the left side, since $\mu_{\ell}<0$. However, in interval $\mathcal{I}_{3}$, every point on the right side is mapped above the fixed point $x^{\mathcal{L}}$. This means that points spend $2 n$ steps, rotating around the swirl on the left side, before they are mapped back to the right side, for some $n \geq 1$. Therefore, we can consider a composite map in which we use $f_{\ell \ell}$ for values $x<0$ and $f_{r}$ for $x>0$. This yields an increasing/decreasing system with both slopes having an absolute value larger than one, as described in $\S 4.4$. The left boundary of interval $\mathcal{I}_{3}$ is given by the homoclinic bifurcations of the fixed point $x^{\mathcal{L}}$ and can be determined as

$$
x^{\mathcal{L}}=f_{r}\left(f_{\ell}\left(f_{\ell}(0)\right)\right) \Rightarrow\left\{\left(a_{\ell}, a_{r}, \mu_{\ell}, \mu_{r}\right) \mid \frac{\mu_{\ell}}{\mu_{r}}=-\frac{a_{\ell}-1}{1+a_{r} a_{\ell}{ }^{2}-a_{r}}\right\}
$$

The right boundary of the interval $\mathcal{I}_{3}$ (according to the conditions given above) represents the border collision of the fixed point $x^{\mathcal{R}}$ at the boundary of the third and fourth quadrants. However, it is likely that the conditions can be relaxed so that the interval $\mathcal{I}_{3}$ ranges up to the homoclinic bifurcation of this fixed point causing the transition from a three-band to a two-band attractor. Indeed, it can be seen in Fig. 15 that a part of interval $\mathcal{I}_{4}$ belongs to the same bandcount incrementing scenario as the one occurring in the interval $\mathcal{I}_{3}$.

(v): In interval $\mathcal{I}_{5}$, defined by the conditions $f_{\ell}\left(f_{\ell}(0)\right)<x^{\mathcal{R}}, x^{\mathcal{L}}<f_{r}\left(f_{r}(0)\right)$ and $\mu_{\ell}<0, \mu_{r}>0$, the chaotic region is structured by the bandcount adding scenario, which is, by contrast to the bifurcations scenarios in the intervals $\mathcal{I}_{1}, \mathcal{I}_{3}, \mathcal{I}_{7}$ and $\mathcal{I}_{9}$ not associated with basic orbits. In fact, this region can be seen as an interior substructure of the overall bandcount incrementing scenario and for other values of $a_{\ell}, a_{r}$ more similar substructures may exist (for details we refer to [25]). In this interval both swirls exist, as $\mu_{\ell}<0$ and $\mu_{r}>0$. Points which leave the swirl on the right side are mapped to the left side, above the fixed point $x^{\mathcal{L}}$. This means that points spend $2 n$ step rotating on the left side, for $n \geq 1$. Behavior on the right side is analogous: Points which leave the left side, are mapped below the fixed point $x^{\mathcal{R}}$, so that they spend $2 n$ steps rotating on the right side, for $n \geq 1$. Therefore, the behavior of the map can be represented by the composite map in which we use $f_{\ell \ell}$ for values $x<0$ and $f_{r r}$ for $x>0$. This way, we get an increasing/increasing system with both slopes larger than one. The absorbing interval of this composite map $f_{\ell \ell} / f_{r r}$ is $\left[f_{r r}(0), f_{\ell \ell}(0)\right]$. The boundaries of interval $\mathcal{I}_{5}$ are given by

$$
\begin{aligned}
& x^{\mathcal{R}}=f_{\ell}\left(f_{\ell}(0)\right) \Rightarrow\left\{\left(a_{\ell}, a_{r}, \mu_{\ell}, \mu_{r}\right) \mid \frac{\mu_{\ell}}{\mu_{r}}=-\frac{1}{\left(a_{r}-1\right)\left(a_{\ell}+1\right)}\right\} \\
& x^{\mathcal{L}}=f_{r}\left(f_{r}(0)\right) \Rightarrow\left\{\left(a_{\ell}, a_{r}, \mu_{\ell}, \mu_{r}\right) \mid \frac{\mu_{\ell}}{\mu_{r}}=-\left(a_{\ell}-1\right)\left(a_{r}+1\right)\right\}
\end{aligned}
$$

They are equal to the collision of the absorbing interval $\left[f_{r r}(0), f_{\ell \ell}(0)\right]$ of the composite map with the unstable fixed points $x^{\mathcal{L}}$ and $x^{\mathcal{R}}$.

(vii) and (ix): The description of the interval $\mathcal{I}_{7}$ can be derived from the description of interval $\mathcal{I}_{3}$ by exchanging each mention of "left" and "right", and by exchanging the symbols $\mathcal{L}$ and $\mathcal{R}$. It's left boundary is equal to the border collision of the fixed point $x^{\mathcal{L}}$ at the boundary between the fourth and first quadrants. It's right boundary is equal to:

$$
x^{\mathcal{R}}=f_{\ell}\left(f_{r}\left(f_{r}(0)\right)\right) \Rightarrow\left\{\left(a_{\ell}, a_{r}, \mu_{\ell}, \mu_{r}\right) \mid \frac{\mu_{\ell}}{\mu_{r}}=-\frac{1+a_{\ell} a_{r}{ }^{2}-a_{\ell}}{a_{r}-1}\right\}
$$

Similarly, the description of interval $\mathcal{I}_{9}$ can be derived from the description of interval $\mathcal{I}_{1}$ by exchanging each mention of "left" and "right", and by exchanging the symbols $\mathcal{L}$ and $\mathcal{R}$. It's left boundary is equal to $\eta_{\ell}$ (Eq. (6)). It's right boundary is equal to $\eta_{r}^{\mathcal{R} L}$ (Eq. (10)). 
Note that the intervals $\mathcal{I}_{1}-\mathcal{I}_{9}$ do not always exist, as Fig. 16 shows. For the parameter values corresponding to these figure, only two intervals exist (denoted as $\mathcal{I}_{3}^{\prime}$ and $\mathcal{I}_{7}^{\prime}$ ) in which the bandcount is not one.

\section{Summary}

In this work we presented an overview over the organizing centers occurring in 2D parameter planes of discontinuous piecewise linear maps. By contrast to previous works we didn't restrict ourselves to the cases where the system function is contracting on both sides of the discontinuity but considered also the cases with slopes with an absolute value larger than one. Following different configurations of the slopes we identified and described seven different cases for all slope configurations which are not conjugate to other cases. Some of these cases are more complex than others, but all of them can be described as a combination of four basic bifurcation scenarios: period adding, period incrementing, bandcount adding and bandcount incrementing, together with the existence regions of fixed points and of the divergent behavior.

If both slopes have an absolute value smaller than one, the decreasing/decreasing case is the least complicated one. We demonstrated that the situation changes, if the absolute values of the slopes are larger than one. In this case, the complexity of the bifurcation structures increases with the number of decreasing slopes with an absolute value larger than one. So the simplest cases are the two increasing/increasing cases, and the increasing/decreasing case, in which only the increasing slope is larger than one. The most complicated case is the decreasing/decreasing case with both slopes smaller than minus one. However, we demonstrated that the more simple cases can be used for descriptions of the more complicated ones, by replacing parts of the system function with iterated versions of itself, according to the periodic orbits relevant for the specific scenario.

The next step in the investigation of piecewise linear maps is to determine analytical conditions for all boundaries between the seven cases. Apart from that, the results of this work can be applied to piecewise-nonlinear systems (for example, piecewise quadratic ones), which should be explored as well.

\section{ACKNOWLEDGMENT}

The authors thank Prof. Laura Gardini from the University of Urbino, Italy, for valuable discussions which significantly improved this work. This work was supported by the DFG project "Organizing centers in nonsmooth dynamical systems: bifurcations of higher codimension in theory and applications".

\section{REFERENCES}

[1] Zh. T. Zhusubaliyev and E. Mosekilde. Bifurcations and Chaos in piecewise-smooth dynamical systems, volume 44 of Nonlinear Science A. World Scientific, 2003.

[2] M. di Bernardo, C. J. Budd, A. R. Champneys, and P. Kowalczyk. Piecewise-smooth Dynamical Systems: Theory and Applications, volume 163 of Applied Mathematical Sciences. Springer, 2008.

[3] D.J.W. Simpson. Bifurcations in piecewise-smooth continuous systems, volume 70 of Nonlinear Science A. World Scientific, 2010.

[4] J. Guckenheimer and R. F. Williams. Structural Stability of Lorenz Attractors. Publ. Math. IHES, 50:307-320, 1979.

[5] J. M. Gambaudo, I. Procaccia, S. Thomae, and C. Tresser. New Universal Scenarios for the Onset of Chaos in Lorenz-Type Flows. Phys. Rev. Lett, 57:925-928, 1986.

[6] D. V. Lyubimov, A. S. Pikovsky, and M. A. Zaks. Universal Scenarios of Transitions to Chaos via Homoclinic Bifurcations, volume 8 of Math. Phys. Rev. Harwood Academic, London, 1989. Russian version 1986 as a Preprint (192) of Russian Academy of Science, Institute of mechanics of solid matter, Sverdlovsk.

[7] V. Avrutin and M. Schanz. On multi-parametric bifurcations in a scalar piecewise-linear map. Nonlinearity, 19:531-552, 2006.

[8] V. Avrutin, M. Schanz, and S. Banerjee. Multi-parametric bifurcations in a piecewise-linear discontinuous map. Nonlinearity, 19:1875-1906, 2006.

[9] V. Avrutin, M. Schanz, and S. Banerjee. Codimension-3 bifurcations: Explanation of the complex 1-, 2- and 3D bifurcation structures in nonsmooth maps. Phys. Rev. E, 75:066205/1-7, 2007.

[10] S. Banerjee, J. A. Yorke, and C. Grebogi. Robust chaos. Phys. Rev. Lett, 80:3049-3052, 1998.

[11] F. Tramontana, L. Gardini, V. Avrutin, and M. Schanz. New Adding Phenomena in Piecewise Linear Maps with two discontinuities. Int. J. Bifurcat. Chaos, 2011. (accepted for publication). 
[12] V. Avrutin, A. Granados, and M. Schanz. Sufficient conditions for a period increment big bang bifurcation in one-dimensional maps. Nonlinearity, 24(9):2575, 2011.

[13] L. Gardini, V. Avrutin, M. Schanz, A. Granados, and I. Sushko. Organizing centers in parameter space of discontinuous 1d maps. the case of increasing / decreasing branches. In Proc. of ECIT 2010, European Series in Applied and Industrial Mathematics, 2011. (to appear).

[14] V. Avrutin, P. Levi, and M. Schanz. Border-collision induced bifurcation phenomena and multi-parametric bifurcations in a piecewise-quadratic map on interval. In Proc. of 5th EUROMECH Nonlinear Dynamics Conf. "ENOC'2005", 2005.

[15] V. Avrutin, L. Gardini, A. Granados, M. Schanz, and I. Sushko. Continuity Breaking and Intersection of Border Collision Bifurcations Curves Leading to Big-Bang Bifurcations. In Proc. of 7th EUROMECH Nonlinear Dynamics Conf. "ENOC'2011", 2011.

[16] P. Jain and S. Banerjee. Border collision bifurcations in one-dimensional discontinuous maps. Int. J. Bifurcation Chaos, 13:3341-3352, 2003.

[17] J. P. Keener. Chaotic behavior in piecewise continuous difference equations. Trans. Am. Math. Soc., 261(2):589-604, 1980.

[18] L. Gardini, F. Tramontana, V. Avrutin, and M. Schanz. Border collision bifurcations in 1D piecewise-linear maps and Leonov's approach. Int. J. Bifurcat. Chaos, 20(10):3085-3104, 2010.

[19] V. Avrutin and M. Schanz. On the fully developed bandcount adding scenario. Nonlinearity, 21:1077-1103, 2008.

[20] V. Avrutin, M. Schanz, and B. Schenke. Coexistence of the bandcount-adding and bandcount-increment scenarios. Discrete Dynamics in Nature and Society, 2011(Article ID 681565), 2011.

[21] V. Avrutin, B. Eckstein, and M. Schanz. The bandcount increment scenario. I: basic structures. Proc. R. Soc. A, 464(2095):1867-1883, 2008.

[22] L. Gardini, I. Sushko, V. Avrutin, and M. Schanz. Critical homoclinic orbits lead to snap-back repellers. Chaos, Solitons $\mathcal{E}$ Fractals, 44:433-449, 2011.

[23] L. Gardini and F. Tramontana. Border collision bifurcation curves and their classification in a family of one-dimensional discontinuous maps. Chaos, Solitons \& Fractals, 44:248259, 2011.

[24] F. Tramontana, F. Westerhoff, and L. Gardini. On the complicated price dynamics of a simple one-dimensional discontinuous financial market model with heterogeneous interacting traders. J. Economic Behavior and Organization, 2011. (to appear).

[25] V. Avrutin, B. Eckstein, and M. Schanz. The bandcount increment scenario. II: interior structures. Proc. R. Soc. A, 464(2097):2247-2263, 2008. 

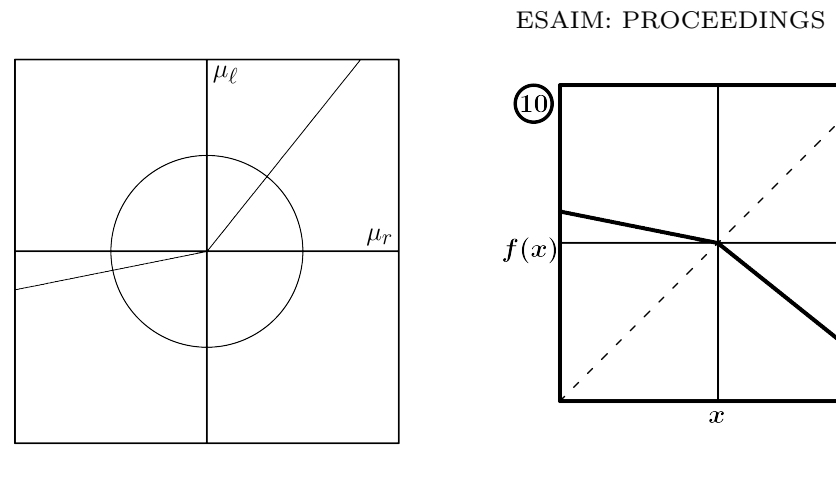

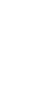
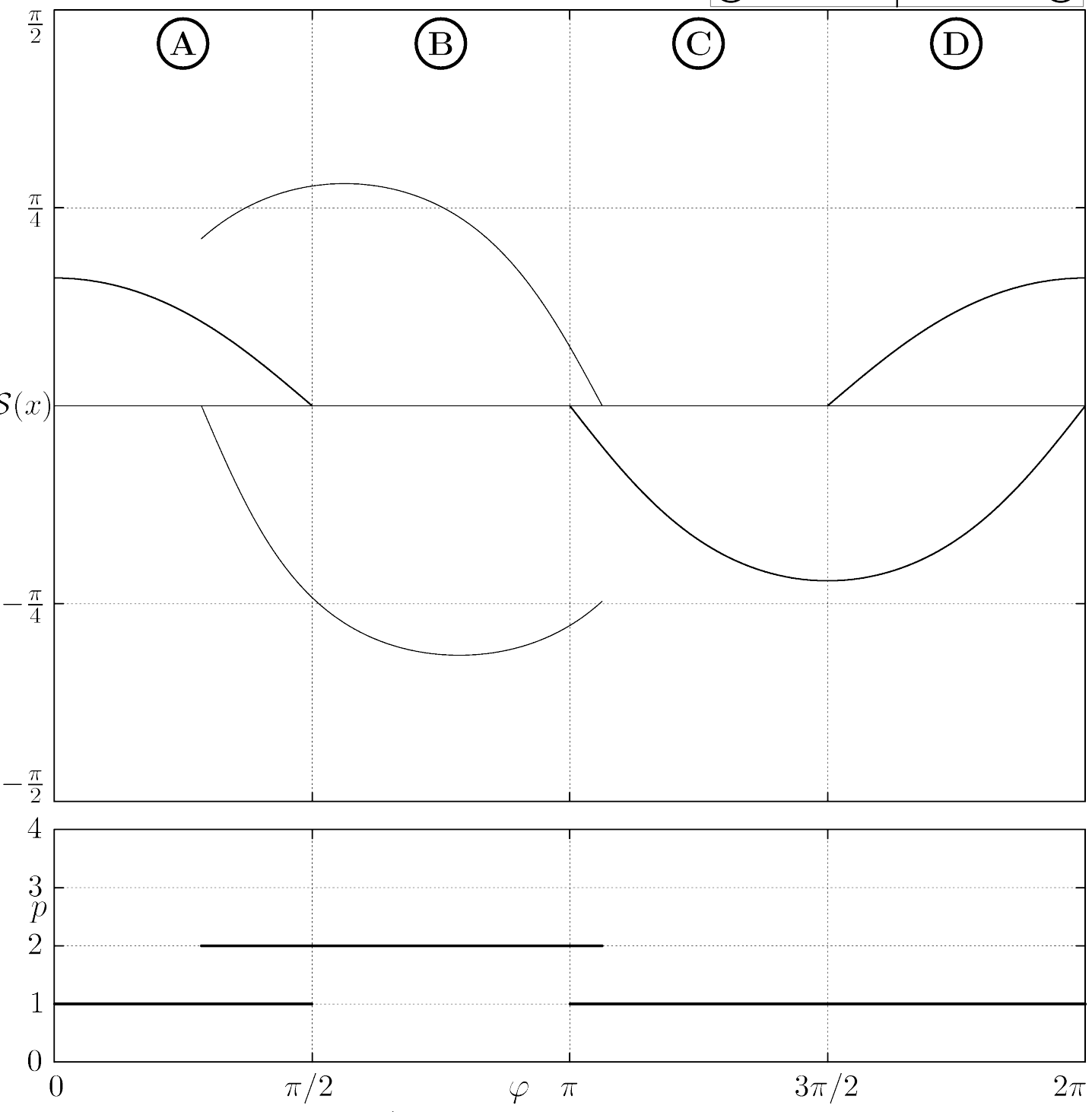

FiguRe 12. Decreasing / decreasing case with $-1<a_{\mathcal{L}}<0,-1<a_{\mathcal{R}}<0$. In the first quadrant, the fixed point $x^{\mathcal{R}}$ and the 2-cycle coexist. The second quadrant contains only the 2-cycle. In the third quadrant, the 2-cycle and the fixed point $x^{\mathcal{L}}$ coexist. In the fourth quadrant $x^{\mathcal{R}}$ and $x^{\mathcal{L}}$ coexist. $\left(a_{\mathcal{L}}=-0.2\right.$, $\left.a_{\mathcal{R}}=-0.8\right)$.
(B)

(A) 101
(C) 


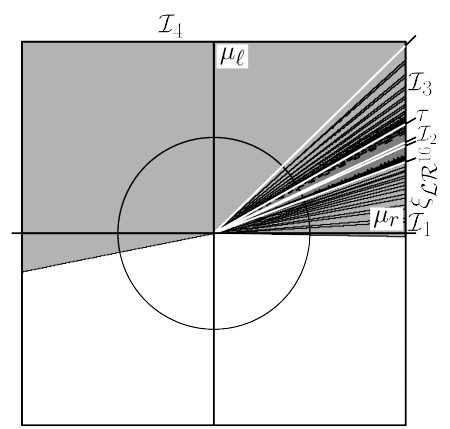

(B)
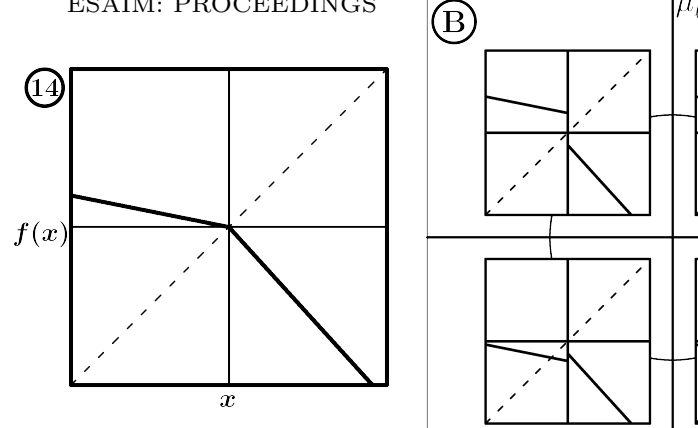

(C)
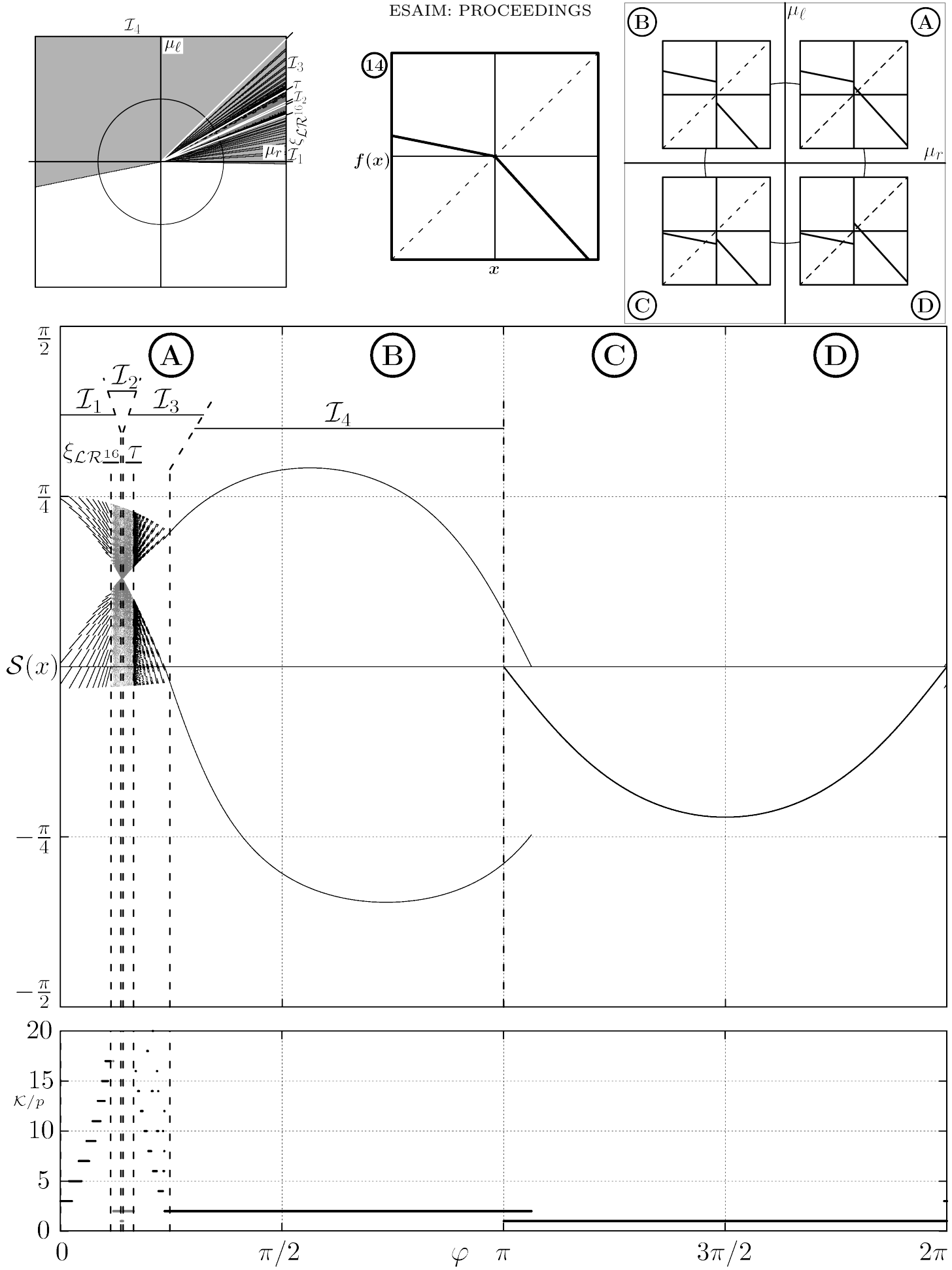

FiguRe 13. Decreasing / decreasing case with $-1<a_{\mathcal{L}}<0, a_{\mathcal{R}}<-1$.

In the first quadrant, there are period incrementing and period adding scenarios, separated by chaotic attractors. The second quadrant contains the 2-cycle, which coexists with $x^{\mathcal{L}}$ in the third quadrant. The fourth quadrant contains $x^{\mathcal{L}}$ and a small piece of period incrementing. $\left(a_{\mathcal{L}}=-0.2, a_{\mathcal{R}}=-1.1\right)$ 

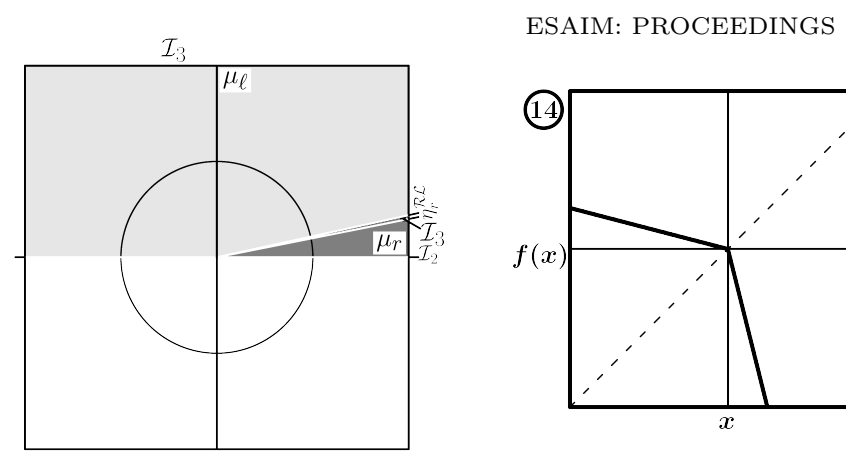

(B)
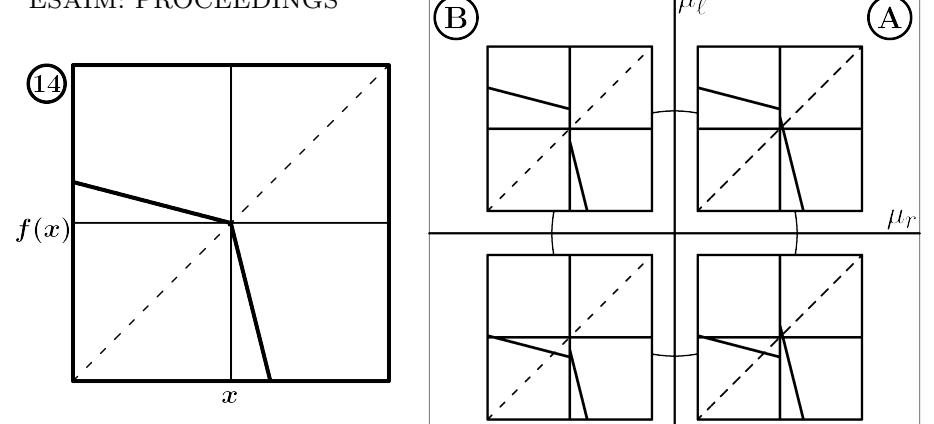

(C)

(D)
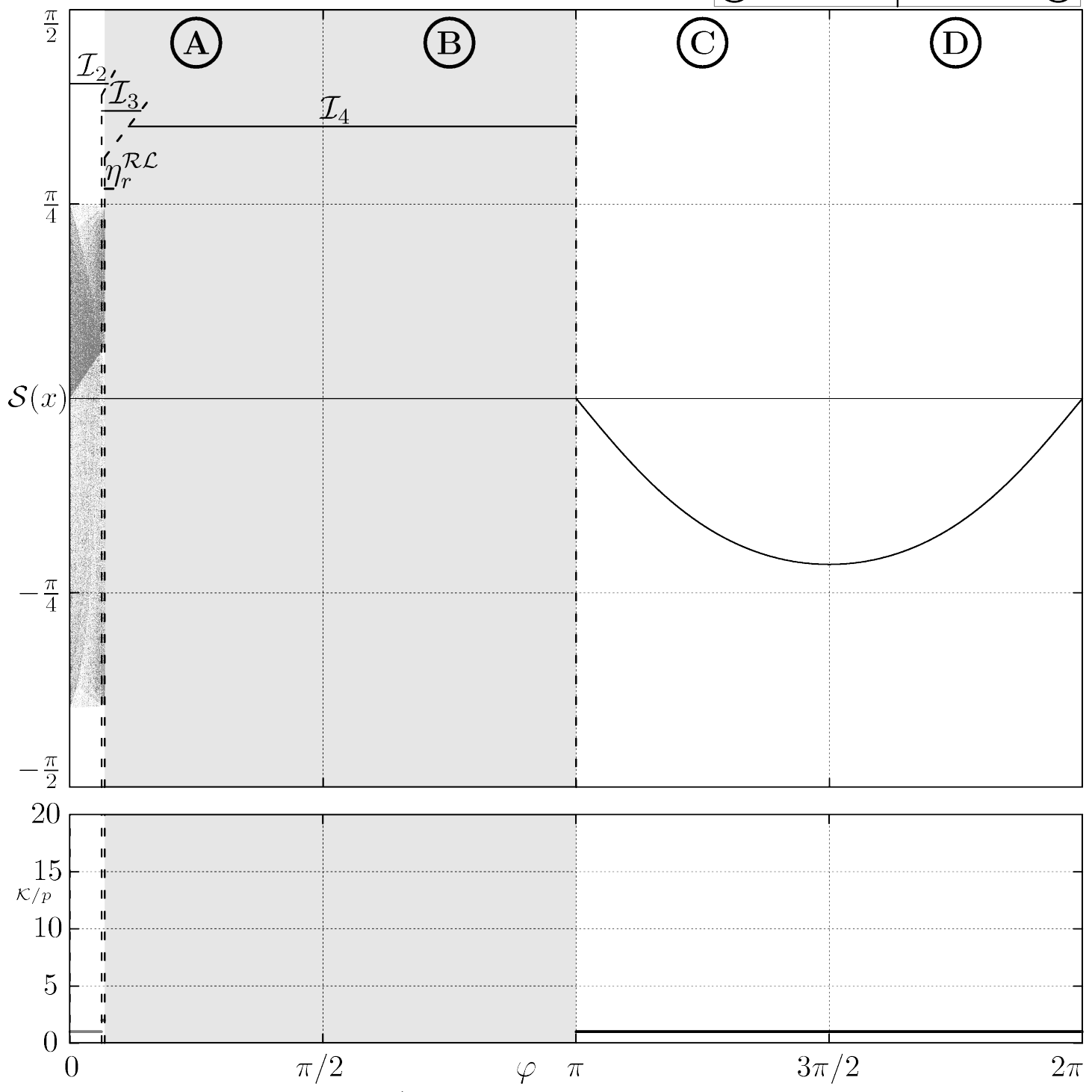

Figure 14. Decreasing / decreasing case with $-1<a_{\mathcal{L}}<0, a_{\mathcal{R}}<-1$.

In the first quadrant are chaotic attractors and divergence. There is only divergence in the second quadrant. The third and fourth quadrants contain the fixed point $x^{\mathcal{L}} .\left(a_{\mathcal{L}}=-0.26, a_{\mathcal{R}}=-4.0\right)$ 

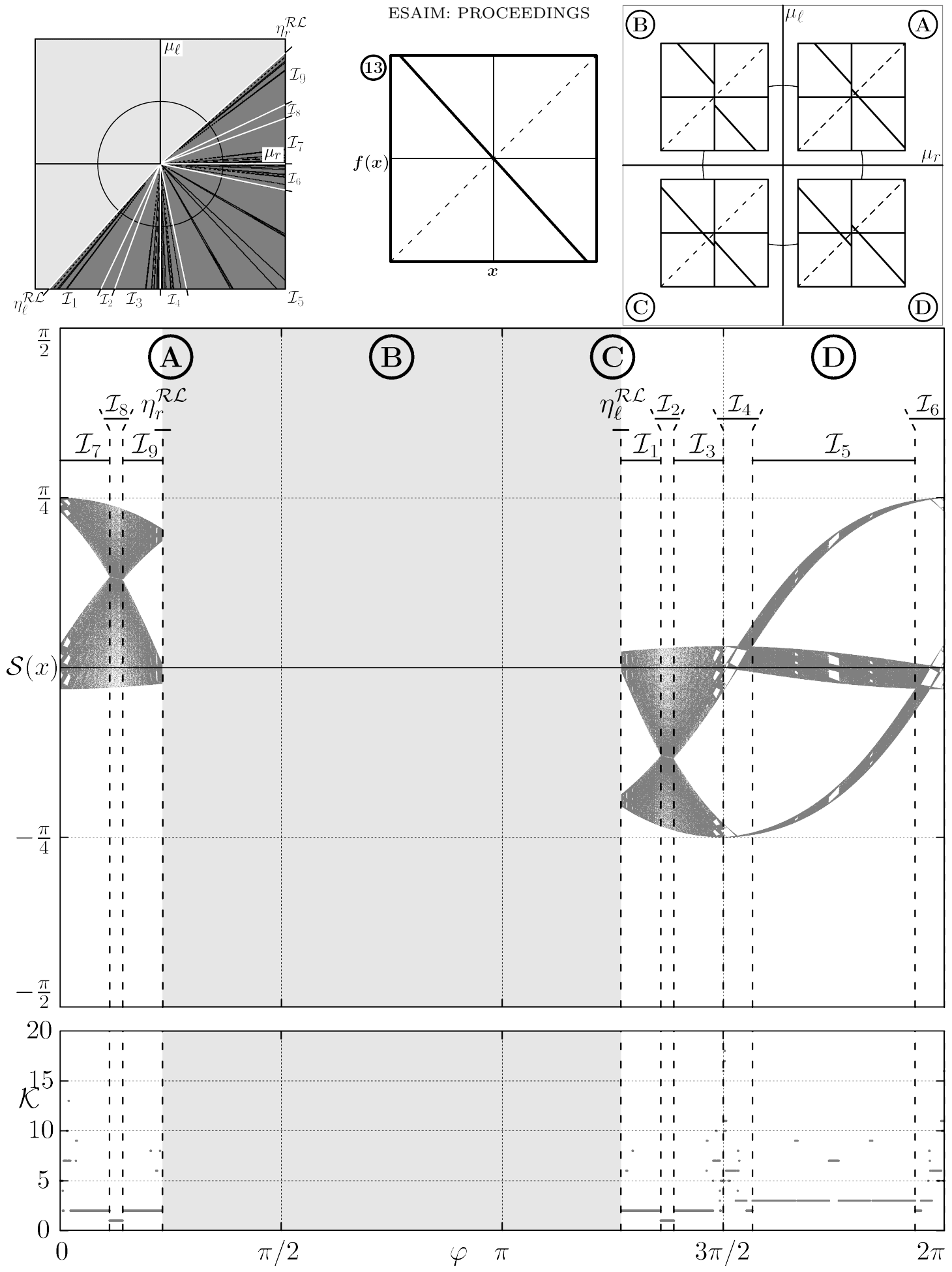

FiguRE 15. Decreasing / decreasing case with $a_{\mathcal{L}}<-1, a_{\mathcal{R}}<-1$.

There is only divergence in the second quadrant, and in parts of the first and third. The first, third and fourth quadrants contain multi-band chaotic attractors structured by bandcount adding and bandcount incrementing scenarios. The structure is symmetric with respect to $\frac{7 \pi}{4}$. $\left(a_{\mathcal{L}}=-1.1, a_{\mathcal{R}}=-1.1\right)$ 


\section{ESAIM: PROCEEDINGS}

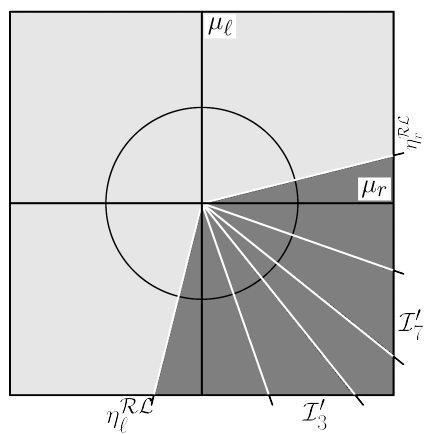

$$
\frac{\pi}{2}
$$

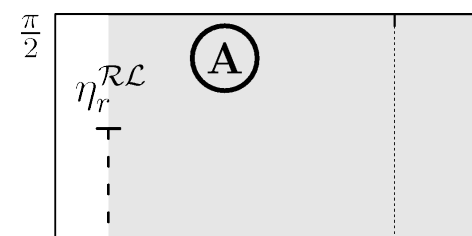

$\frac{\pi}{4}$
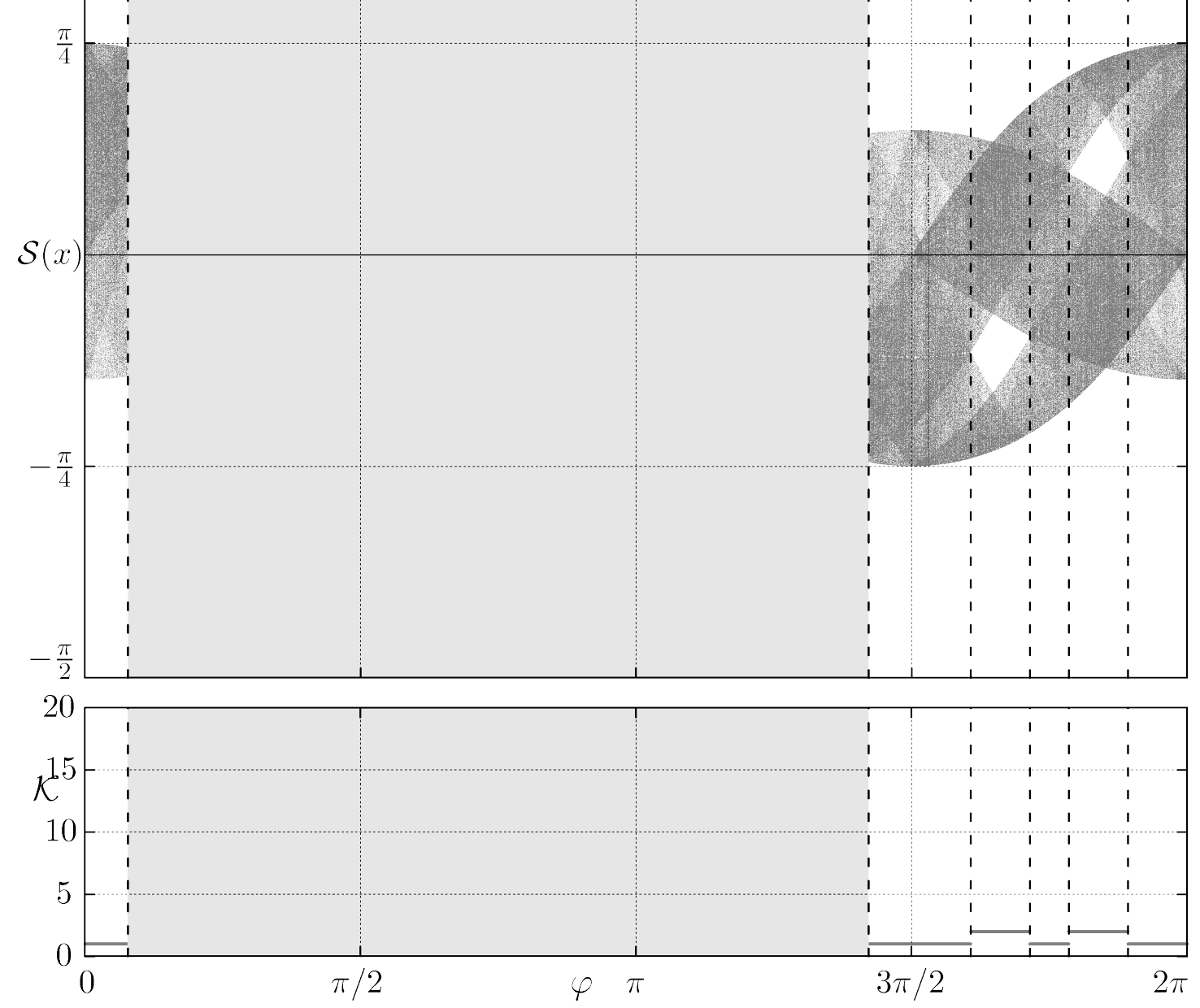

FIGURE 16. Decreasing / decreasing case with $a_{\mathcal{L}}<-1, a_{\mathcal{R}}<-1$.

There is only divergence in the second quadrant, and in parts of the first and third. The first, third and fourth quadrants contain chaotic attractors with one or two bands. The structure is symmetric with respect to $\frac{7 \pi}{4}$. $\left(a_{\mathcal{L}}=-1.5\right.$, $\left.a_{\mathcal{R}}=-1.5\right)$ 